\title{
Développement territorial autonome ou assisté
}

Quelle transition fiscale pour la Nouvelle-Calédonie?

\section{Gaël Lagadec}

\section{(2) OpenEdition}

1 Journals

\section{Édition électronique}

URL : http://journals.openedition.org/developpementdurable/3273

DOI : 10.4000/developpementdurable.3273

ISSN : 1772-9971

Éditeur

Association DD\&T

\section{Référence électronique}

Gaël Lagadec, « Développement territorial autonome ou assisté », Développement durable et territoires [En ligne], Varia (2004-2010), mis en ligne le 09 novembre 2010, consulté le 10 décembre 2020. URL http://journals.openedition.org/developpementdurable/3273; DOI : https://doi.org/10.4000/ developpementdurable.3273

Ce document a été généré automatiquement le 10 décembre 2020.

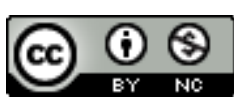

Développement Durable et Territoires est mis à disposition selon les termes de la licence Creative Commons Attribution - Pas d'Utilisation Commerciale 4.0 International. 


\section{Développement territorial autonome ou assisté}

Quelle transition fiscale pour la Nouvelle-Calédonie ?'

\section{Gaël Lagadec}

1 La France a abandonné progressivement son empire colonial à partir des années 1960, pour ne conserver qu'un petit nombre de territoires rattachés à la métropole. Aujourd'hui, il ne subsiste plus que les quatre Départements d'Outre Mer (DOM) traditionnels, devenus des Départements et Régions d'Outre-Mer; les Collectivités d'Outre-Mer (COM), appellation générique qui englobe, depuis la révision constitutionnelle de 2003, deux des trois anciens TOM (la Polynésie française et Wallis-etFutuna) et les deux collectivités particulières de Mayotte et de St-Pierre-et-Miquelon, auxquels pourraient s'adjoindre à terme Saint Barthélemy et Saint Martin, jusque-là rattachés administrativement à la Guadeloupe mais qui ont souhaité devenir autonomes ; les Terres Australes et Antarctiques Françaises (dont on peut se demander si leur futur statut n'en fait pas une catégorie à part); enfin, la Nouvelle-Calédonie, régie par un titre spécifique de la Constitution française.

2 La situation de la Nouvelle-Calédonie, COM du Pacifique Sud, d'environ 240000 habitants (ISEE 2007a. L'ISEE est L'institut (calédonien) de la Statistique et des Études Économiques), est très particulière, puisqu'il s'agit de la seule collectivité inscrite dans un processus d'émancipation vis-à-vis de la Métropole ${ }^{2}$. À bien des égards la NouvelleCalédonie correspond à un pays en développement. Si ses caractéristiques socioéconomiques sont largement celles d'un pays développé En effet, l'Indice de Développement Humain pour la Nouvelle-Calédonie est estimé à 0,87(CEROM, (2005); l'acronyme CEROM signifie Comptes Économiques Rapides de l'Outre Mer), ce qui la place en $32^{\text {ème }}$ place sur 177 pays $^{3}$. D'autres caractéristiques sont plutôt celles d'un pays en développement : inégalités de revenus, concentration des activités industrielles autour de la seule grande agglomération, secteur exportateur quasiment mono producteur.

3 Le dualisme typique du tiers-monde se retrouve jusque dans le découpage provincial, puisque la Nouvelle-Calédonie compte une province Sud développée et englobant Nouméa 
et des provinces Nord et Iles particulièrement pauvres. La monnaie ayant cours légal en Nouvelle-Calédonie, comme dans les autres collectivités d'outre-mer du Pacifique (Polynésie française et Wallis-et-Futuna), est le Franc CFP. Il bénéfice, depuis 1949, d'une parité fixe avec le Franc métropolitain (parité inchangée avec le passage à l'euro4). En 2008, le produit intérieur brut du territoire était de 728 milliards de F. CFP soit environ 6 milliards d'euros. Les ressources propres au territoire sont les produits miniers, qui représentent plus de $90 \%$ des exportations (mais moins de $10 \%$ du PIB). À cela s'ajoutent les transferts en provenance de la métropole qui correspondent depuis le début des années quatre-vingt-dix au quart du PIB calédonien ${ }^{5}$. La ressource minière ne signifie cependant pas richesse, ni garantie de développement (CEROM, 2005, pp. 17-18, pour une présentation des manifestations $d u$ « syndrome hollandais » en Nouvelle-Calédonie).

Le retard de développement peut être stigmatisé par la fiscalité calédonienne, puisque la Nouvelle-Calédonie est l'un des rares territoires à ne pas encore disposer d'une TVA. Cette particularité se retrouve dans l'organisation du commerce extérieur. Les taxes douanières jouent un rôle d'autant plus important que le territoire ne dispose pas de l'outil fiscal de la TVA. Le taux de couverture des importations par les exportations se situe à un niveau faible, oscillant, selon les années, autour de $50 \%$. Ce sont naturellement les transferts de la métropole qui desserrent la contrainte extérieure. Cette situation de dépendance est largement paradoxale, considérant que la Nouvelle-Calédonie est inscrite dans un processus d'émancipation. Dépendance économique et émancipation semblent antinomiques, a fortiori, en considérant que les transferts n'ont pas de raison de conserver leur niveau dans l'optique de l'émancipation (et encore moins en considérant les difficultés budgétaires structurelles de l'État français). Le contexte politique étant inscrit dans une loi et son issue dépendant d'un choix électoral relativement lointain, c'est l'action sur l'environnement économique qui peut éventuellement résoudre l'antinomie.

Nous proposons ici d'explorer la soutenabilité du système économique actuel néocalédonien, reposant sur des transferts métropolitains voués à diminuer. La soutenabilité est ici entendue sous un angle économique et temporel et non pas environnemental: comment le système économique peut-il perdurer sans connaître de crise structurelle? C'est en ce sens que la TVA est un enjeu particulier. Dans le contexte de l'émancipation et de la mise en conformité avec les principes de l'Organisation Mondiale du Commerce (OMC), la Nouvelle-Calédonie devra s'insérer dans le commerce international. Elle ne pourra conserver son système douanier actuel, qui finance les collectivités locales à partir (essentiellement) d'importations en provenance de la métropole, indirectement financées par les transferts métropolitains. La réforme fiscale devra alors être la substitution d'une fiscalité dynamique et incitative à une fiscalité protectionniste confiscatoire ${ }^{6}$.

Dans une première section nous détaillons les justifications de la réforme fiscale à partir des enseignements de la fiscalité optimale et des contraintes internationales susceptibles de s'imposer à la Nouvelle-Calédonie. Dans une deuxième section, nous décrivons le système commercial calédonien et les relations Calédonie-Métropole. Dans les troisième et quatrième sections nous présentons les principes généraux de la TVA et de la TVA "sociale", avant d'étudier (cinquième section) les possibilités d'améliorer l'environnement économique en Nouvelle-Calédonie par une voie fiscale, en substituant une TVA à la fiscalité douanière (TVA fiscale ou TVA sociale). 


\section{Pourquoi réformer la fiscalité ?}

\subsection{Fiscalité directe ou indirecte : les enseignements de la fiscalité optimale}

7 Les impôts directs sont ceux qui peuvent être appliqués aux agents en fonction de leurs caractéristiques économiques et sociales. Ces impôts peuvent avoir un taux non linéaire et permettent ainsi la progressivité. Les impôts indirects sont au contraire basés sur des variables non observables au niveau individuel (transactions anonymes), ce qui ne permet qu'une taxation linéaire. La problématique de la fiscalité optimale est celle de l'arbitrage entre efficacité et équité (redistribution) quand l'impôt doit s'appliquer à des situations individuelles dont les caractéristiques ne sont pas observables (donc essentiellement en fiscalité indirecte). Cet arbitrage amène des taux d'imposition différenciés; c'est en cela que des taux supérieurs doivent s'appliquer aux biens ayant pour caractéristiques une faible élasticité prix en valeur absolue (i) et une forte élasticité revenu (ii). La première caractéristique (i) vise un objectif d'efficacité. Plus l'élasticité prix est faible (en valeur absolue) et moins la taxe sera distorsive (faible effet de substitution). Les principes de la fiscalité douanière sont d'ailleurs conformes à ce résultat. La seconde caractéristique (ii) vise un objectif d'équité : en taxant les biens majoritairement consommés par les ménages à hauts revenus, on permet la redistribution.

8 Ces deux objectifs s'opposent pour les biens inférieurs et les biens de luxe. Les biens inférieurs ont une faible élasticité prix, mais (par définition) également une élasticité revenu négative. Les biens de luxe ont (par définition) une forte élasticité revenu mais peuvent également avoir une élasticité prix fortement négative. Pour ces biens, ces deux effets s'opposent donc mais (sauf cas particulier) sans se neutraliser. Ils limitent donc la différenciation des taux sans la supprimer.

Dans cette optique (et dans un cadre statique), A. B. Atkinson et J. E. Stiglitz (1976) montrent que l'impôt indirect est dominé par l'impôt direct, c'est-à-dire qu'il vaut toujours mieux taxer les revenus que les biens. Ce résultat peut être expliqué ainsi : les agents ont des rémunérations différentes, découlant de l'hétérogénéité de leurs productivités ; l'impôt sur le revenu demeure (sous la contrainte de ne pas décourager l'offre de travail) le meilleur instrument pour corriger ces inégalités (Cremer 1999, pp. 86-87). Cela signifie notamment qu'une détermination optimale de l'impôt sur le revenu l'emportera toujours sur une TVA à taux différenciés (à recette constante l'objectif d'équité est moins satisfait par la TVA, taxation de la consommation; si on considère une TVA à taux unique elle est alors simplement équivalente à un impôt sur le revenu proportionnel).

10 Ce résultat négatif pour les impôts indirects est toutefois remis en question dès lors qu'on relâche les hypothèses du modèle concernant l'aspect temporel (i) et les productivités individuelles (ii).

11 (i) Si l'on abandonne le cadre statique, l'équivalence susmentionnée entre impôt sur la consommation et impôt sur le revenu ne tient plus. À taux (uniformes) égaux, la pression fiscale est supérieure dans le cadre d'un impôt sur le revenu, du fait d'une double taxation de la part du revenu non immédiatement consommée. L'épargne est en effet taxée deux fois : une première fois au titre du revenu (qui sera en partie épargné) et une seconde fois au titre des revenus de l'épargne. A contrario, la consommation n'est taxée 
qu'une seule fois. La consommation différée est donc plus fortement taxée que la consommation présente. Cette différence n'a pas de justification au regard des objectifs de la fiscalité optimale : consommations présente et future ne peuvent être discriminées selon des critères d'élasticité prix ou revenu. Les principes de la fiscalité optimale peuvent donc amener à conclure que, dans un cadre dynamique, il est préférable (pour éviter la double taxation de l'épargne) de financer les recettes fiscales par un impôt sur la consommation (de type TVA) plutôt que par un impôt sur le revenu.

(ii) La supériorité de l'imposition directe sur l'imposition indirecte ne vaut également que tant que les rémunérations (via les productivités individuelles) ne sont que la seule source d'hétérogénéité. Si on fait l'hypothèse que les richesses des individus divergent au-delà de leurs revenus respectifs (dotations initiales), alors la consommation n'est plus seulement fonction du revenu. Dans ce cas, des impôts indirects peuvent utilement compléter les impôts directs selon l'objectif de redistribution. Par exemple, des taux de TVA majorés sur les biens de luxe permettront de discriminer entre les agents riches et les autres même si les transactions individuelles ne sont pas observables. Or, il apparaît (dans le cas français) que non seulement l'hétérogénéité des agents est forte en matière de patrimoine, mais qu'elle est même nettement supérieure à l'hétérogénéité en matière de revenus (Houriez et Roux 2001, p. 278).

Réformer la fiscalité calédonienne en substituant une TVA à une fiscalité douanière ne heurte donc pas de prescriptions d'un point de vue théorique (dès lors qu'on raisonne sur plusieurs périodes, l'imposition directe ne domine pas l'imposition indirecte selon les principes de la fiscalité optimale).

14 Cette réforme est par ailleurs une réforme de la fiscalité indirecte, qui coexiste partout avec une fiscalité directe, pour des raisons tant économiques que politiques. La fiscalité directe ne dominant pas automatiquement la fiscalité indirecte, l'introduction de la TVA sera désirable, dès lors qu'elle sera considérée comme préférable à la fiscalité douanière.

\subsection{Les avantages de la TVA}

15 En pratique, il semble que la TVA ait un impact redistributif très faible. La raison en est que la consommation des ménages n'est pas suffisamment différenciée selon le niveau de revenu (ou de richesse). Ainsi, par rapport au revenu, la TVA payée par les ménages français varie très peu selon les déciles (Lamotte et Saint Aubin 1999, pp.110-111 ou Besson 2007, p. 42). L'aspect redistributif est notamment limité par le fait que les biens de luxe (livres, produits de la culture) sont faiblement taxés pour des raisons non économiques (promouvoir l'accès à la culture) alors qu'ils sont majoritairement consommés par les ménages à hauts revenus. Toutefois, quand on aborde une perspective dynamique, la TVA apparaît plus redistributive. Dans ce cadre l'effet direct anti redistributif d'un relèvement de la TVA est contrebalancé, d'une part, par l'effet de l'indexation du salaire minimum et des revenus de transferts sur l'inflation; d'autre part, par les comportements de consommation qui modifient la structure des dépenses au détriment des biens qui se renchérissent le plus (Besson 2007, p. 47).

Dans le cadre de la Nouvelle-Calédonie, la TVA présente deux types d'avantages: des avantages généraux (qui recoupent la problématique des réformes dans les pays en développement - PED) et des avantages plus spécifiques, concernant le remplacement d'un ensemble de taxes diverses par une TVA. 


\subsubsection{Des avantages généraux} une fiscalité moderne à la fiscalité douanière. Cette nécessité a deux causes liées : (i) se mettre à terme en conformité avec les principes de $\mathrm{l}^{1} \mathrm{OMC}^{7}$; (ii) dans le cadre de l'émancipation et donc de la baisse des transferts métropolitains, substituer des ressources de fiscalité interne à des recettes tarifaires décroissantes, afin de mettre en place un mode de financement pérenne des biens publics.

(i) L'Accord Général sur les Tarifs Douaniers et le Commerce (GATT) de 1947, ainsi que celui de 1994, ont été acceptés par la France sans réserve d'application territoriale. Les règles de l'OMC en matière d'importation des marchandises ont donc vocation à s'appliquer non seulement au territoire douanier métropolitain, mais également aux territoires douaniers de l'ensemble de ses collectivités territoriales. Cette interprétation est renforcée à la lecture de la réponse du Ministre de l'économie et des finances à $\mathrm{E}$. Vernaudon, député de la Polynésie française, publiée au JORF du 31 mai 1999, et dans laquelle il est indiqué que "l'Accord de Marrakech instituant l'OMC s'applique à l'ensemble des collectivités territoriales d'outre-mer français [...] ». Il semble donc que les Accords de l'OMC en matière de commerce des marchandises s'appliquent en Nouvelle-Calédonie et que la Nouvelle-Calédonie soit tenue au respect des dispositions de ces instruments lors de l'élaboration de son tarif douanier. Concernant les barrières non tarifaires, il apparait clairement qu'elles sont illégales au regard de l'OMC ${ }^{8}$.

(ii) Le passage d'une fiscalité douanière à une TVA est une problématique très proche de celle de la transition fiscale dans les PED, notamment d'Afrique subsaharienne. "Entre 1980-1982 et 2000-2002 est intervenue, à la suite des politiques de libéralisation commerciale, pour l'ensemble des PED (...) une baisse remarquable de la dépendance des ressources publiques vis-à-vis des taxes sur le commerce extérieur. (...) Cependant, en Afrique, les taxes sur le commerce extérieur contribuent encore à $22 \%$ des recettes publiques contre environ $10 \%$ pour les autres PED.» (Chambas 2005, pp. 136-137). En Nouvelle-Calédonie, la part des taxes douanières dans le total des taxes directes et indirectes s'élève à $33 \%$ (Descombels 2007, p. 141), ce qui témoigne du décalage du système fiscal calédonien vis-à-vis de la fiscalité dans les PED et a fortiori dans les pays développés. "Des mutations radicales sont en cours en Afrique comme dans les autres PED. Les pays africains ont déjà réduits de manière substantielle leurs tarifs douaniers et sont désormais engagés dans une transition fiscale dont la TVA est le pivot; un grand nombre de pays doivent faire progresser leur niveau de ressources publiques. Une des conditions (est) de poursuivre l'adaptation des systèmes fiscaux et douaniers. " (Chambas 2005, p. 141). M. Keen et J. L. Ligthart (2002) étudient la question de la transition fiscale (substitution des tarifs par une TVA) à travers l'objectif de stabilité des recettes fiscales pendant la transition. Ils démontrent qu'une stratégie simple pour cela consiste à compenser point par point la réduction des tarifs par une hausse des taxes à la consommation afin de laisser les prix à la consommation inchangés. Pour une petite économie ouverte, une telle stratégie de réformes coordonnées augmente les recettes fiscales et le bien-être social, tant que la réforme douanière augmente la productivité (Keen et Ligthart 2002, p. 491, p. 493 et p. 504). Les auteurs montrent par la suite que ces résultats peuvent parfois être remis en cause en présence de pouvoirs de marché (Keen et Ligthart 2005, p. 389).

Développement durable et territoires , Varia (2004-2010) | 2010 


\subsubsection{Des avantages spécifiques} multitude de taxes spécifiques, en faveur d'une taxe indifférenciée devra avoir un impact à la baisse sur le prix. Ainsi, selon C. Carbonnier : «les taxes spécifiques entraînent de plus importantes hausses de prix que les taxes ad valorem. Ainsi, passer d'une taxation spécifique à une taxation ad valorem permet, soit de baisser les prix à recettes publiques constantes, soit d'augmenter les recettes publiques à prix constants.» (Carbonnier 2006, p. 62). En outre, la multiplicité des taxes pose le problème de l'instabilité du taux d'imposition moyen. Selon les recommandations de la doctrine du "tax smoothing", il vaut mieux un taux de taxation constant plutôt qu'un taux variable (même si les variations se compensent dans le temps). Et pour cela il vaut mieux une "grande » taxe qu'un ensemble de "petites » taxes. Cette recommandation vaut notamment dans le cadre de la gestion de la dette publique $^{9}$, qui n'est pas le plus pertinent pour la Nouvelle-Calédonie. Mais, plus largement, la multiplicité des taux et la variabilité du taux moyen sont sources d'incertitude, défavorable à la croissance économique. Ainsi, «Comme les inefficiences dans l'économie croissent disproportionnément par rapport à l'augmentation des impôts, le lissage des taux d'imposition d'une année à l'autre peut contribuer largement à renforcer les incitations à la croissance économique. » (Ulla 2006). Une TVA en lieu et place d'un ensemble de "petites » taxes est, sinon une garantie, au moins un avantage dans l'optique d'une fiscalité constante dans le temps.

\section{La structure du commerce extérieur calédonien}

\subsection{Un système protectionniste}

Depuis le $1^{\mathrm{er}}$ janvier 2000, la Nouvelle-Calédonie est compétente pour ce qui est de la réglementation du commerce extérieur. Historiquement, les principales taxes sur les importations calédoniennes (celles qui concernent un maximum de produits) sont les droits de douanes et la Taxe Générale à l'Importation (TGI). Ces taxes ont été modifiées par la réforme de la fiscalité douanière en 2000 (réforme applicable au $1^{\mathrm{er}}$ septembre 2000), qui en a également rajouté et supprimé ${ }^{10}$. Antérieurement à la réforme de 2000, le droit de douanes correspondait à douze taux allant de 0 à $20 \%$ et s'appliquant à tous les produits importés originaires des pays autres que les États membres de l'Union européenne et les DOM, les TOM associés à la Communauté et les États africains signataires de la convention de Yaoundé. La TGI (taxe spécifiquement calédonienne qui s'applique à tous les produits) possédait cinq taux qui variaient de 0 à $41 \%$ : le taux super réduit s'élevait à $6 \%$, le taux réduit à $12 \%$, le taux normal à $24 \%$, le taux intermédiaire à $31 \%$, le taux majoré à $41 \%$.

22 La réforme de 2000 a ramené à cinq les taux des droits de douanes (qui s'appliquent toujours aux biens provenant des mêmes pays) : $0 \%, 5 \%, 10 \%, 15 \%, 20 \%$ (fixés par le tarif des douanes). En revanche, la réforme a étendu les taux de la TGI, qui sont maintenant les suivants : le taux exempt ( $0 \%$ ) s'applique aux biens de première nécessité, matières premières et emballages, biens d'équipement et de production, produits de l'art et de la culture. Le taux alimentaire de niveau 1 (4\%) s'applique aux biens alimentaires considérés comme sociaux. Le taux alimentaire de niveau $2(11 \%)$ s'applique à la majorité des produits alimentaires. Le taux alimentaire de niveau $3(26 \%)$ s'applique aux produits à

Développement durable et territoires, Varia (2004-2010) | 2010 
«fort achat d'impulsion». Le taux anti-évasion (11\%) s'applique aux produits souvent achetés hors du territoire. Le taux des produits culturels et sportifs (11\%) s'applique aux produits correspondants. Le taux normal (21\%) s'applique aux biens non alimentaires courants. Le taux majoré s'applique aux produits de luxe. (Source : ITSEE 2000, p. 251.)

La Taxe de Base à l'Importation (TBI) est perçue sur la quasi-totalité des marchandises au taux de $5 \%$. Quelques produits en sont exemptés (riz, lait, blé, vaccins, livres, engins agricoles et bateaux de pêche). La Taxe de Consommation Intérieure frappe, à des taux variables, les produits habituellement surtaxés. La TCPPL comprend des taux de 4 à $60 \%$ et s'applique aux biens produits localement. La Taxe sur le Fret Aérien (TFA), frappe à un taux de $8 \%$ les marchandises importées par voie aérienne. La Taxe de péage frappe à un taux de $1 \%$ les marchandises importées par voie maritime.

Bien que ne concernant que peu de biens, la taxe la plus intéressante pour une analyse en protection endogène est la Taxe Conjoncturelle de Protection de la Production Locale (TCPPL), puisqu'elle vise explicitement à protéger l'industrie locale. La protection accordée à ces biens correspond donc à des cas typiques de protection commerciale endogène. Sur les quelques dizaines de types de produits concernés par la Taxe Conjoncturelle pour la Protection de la Production Locale, seul un secteur correspond à une grande entreprise : la Société Le Froid (avec plus de cent employés), notamment pour la bière (Voir ITSEE 2000 (p. 175) pour la liste des entreprises calédoniennes comprenant plus de 60 salariés).

Les protections élevées se concentrant sur quelques petites entreprises, cela démontre que la protection commerciale a peu à voir avec un souci de protection-préservation de l'emploi local (voir Perret 2002, chapitre 3, pour une revue des arguments traditionnels de la protection et leur aspect souvent fallacieux), d'autant plus que certains des biens taxés étant des biens alimentaires, il s'agit donc des cas les plus néfastes en termes de bien-être et d'équité sociale puisqu'ils détériorent d'abord la situation des ménages les plus modestes. Ces cas sont d'ailleurs en contradiction avec l'orientation générale de la réforme de la TGI, qui exempte ou taxe à 4 ou $11 \%$ les biens alimentaires - ils ne sont donc explicitement pas guidés par la recherche du bien-être collectif. En outre dans de nombreux cas, la TCPPL s'accompagne d'une protection quantitative (quota ou interdiction). Les tableaux et graphiques suivants donnent un aperçu en chiffres du commerce extérieur calédonien, et de la structure de ses taxes aux importations.

\subsection{Aperçu en chiffres}

Tableau 1. L'évolution du commerce calédonien depuis 2000

\begin{tabular}{|c|c|c|c|c|c|c|c|c|c|}
\hline & 2000 & 2001 & 2002 & 2003 & 2004 & 2005 & 2006 & 2007 & 2008 \\
\hline Exportations & 74184 & 59609 & 62477 & 82759 & 98860 & 104438 & 127923 & 18388 & 110370 \\
\hline Importations & 119007 & 124420 & 127174 & 165196 & 156545 & 170692 & 200826 & 244096 & 262188 \\
\hline Tx de couv. & $62 \%$ & $48 \%$ & $49 \%$ & $50 \%$ & $63 \%$ & $61 \%$ & $64 \%$ & $75 \%$ & $42 \%$ \\
\hline
\end{tabular}

Unités : millions de F. CFP, \% ; Source : ISEE (www.isee.nc).

L'annexe 2 présente le graphique l'évolution des échanges et du taux de couverture de la Nouvelle-Calédonie. Le tableau 2 expose la répartition des recettes fiscales par taxes et indique les taxes dont la substitution par une TVA est envisagée. 
Tableau 2. La répartition des recettes fiscales par taxes en 2006 et 2007

\begin{tabular}{|c|c|c|c|}
\hline Taxes et impôts indirects & $\begin{array}{c}\text { Montaut } \\
2006\end{array}$ & $\begin{array}{l}\text { Moutant } \\
2007\end{array}$ & $\begin{array}{l}\text { Substit. Par } \\
\text { TVA }\end{array}$ \\
\hline Taxe générale à l'importation & 14915 & 15274 & Prévie \\
\hline Taxe de solidarité sur les services & 9098 & 11406 & Prévue \\
\hline Droits d'enregistrement & 6849 & 7998 & \\
\hline Taxe de base à l'importation & 5901 & 6213 & Prévue \\
\hline Droits de douane à l'importation & 4685 & 5304 & \\
\hline Taxe sur les produits pétroliers & 3124 & 4875 & \\
\hline Taxe de consonmation sur les produits importés & 2293 & 1445 & \\
\hline Taxe sur les spectacles et les jeux & 2275 & 2506 & \\
\hline Taxe sur les alcools et tabacs en faveur du secteur sanitaire et social & 1956 & 1959 & \\
\hline Taxe sur le fret aérien & 1583 & 1712 & Prévule \\
\hline Produit de la vente des vignettes pour véhicules automobiles & 1013 & 1130 & \\
\hline Taxe conjoncturelle de protection des productions locales & 805 & 841 & \\
\hline Taxe sur les assurances & 605 & 697 & \\
\hline Taxe de soutien aux productions agricoles et agro-alimentaires & 594 & 758 & \\
\hline Taxe sur l'electricité & 349 & 595 & \\
\hline Taxe de péage & 253 & 281 & Prévue \\
\hline Autres impóts indirects & 1453 & 3430 & \\
\hline Total & 57751 & 66424 & \\
\hline Total des taxes douanières & 28599 & 29077 & \\
\hline \multicolumn{4}{|l|}{ Taxes et impóts directs } \\
\hline Impôt sur les sociétés & 22811 & 41648 & \\
\hline Impót sur le revenu des personnes physiques & 12764 & 13024 & \\
\hline Contribution des patentes & 4965 & 5525 & Prévue \\
\hline Impôt sur le revenu des valeurs mobilières & 4026 & 4.357 & \\
\hline Contribution téléphonique & 1492 & 1591 & \\
\hline Contribution foncière & 1134 & 1211 & \\
\hline Autres taxes directes & 4174 & 6094 & \\
\hline Total & 51366 & 73450 & 40411 \\
\hline Total général & 109117 & 139874 & \\
\hline Paut des taxes douanières & $26 \%$ & $21 \%$ & \\
\hline
\end{tabular}

Unité : millions de F.CFP ; Source : ISEE(2007b, p. 100 et ISEE 2008, p. 100

On constate que les trois principales taxes représentent près de $80 \%$ des recettes douanières (les autres taxes sont des taxes spécifiques). Ces taxes sont précisément des outils de collecte, dans le sens où elles ne visent pas spécifiquement une protection de la production locale. A contrario, la Taxe Conjoncturelle sur la Protection de la Production Locale ne représente que 1,3\% du total des taxes et impôts indirects. L'objectif de collecte fiscale se retrouve quand on étudie la part des recettes douanières dans les recettes du territoire, qui s'établit à $33 \%$.

Le ratio taxes sur valeurs des importations est également intéressant. En 2005, il était légèrement supérieur à $20 \%{ }^{11}$. Le tableau suivant donne des éléments de comparaison (Laborde 2007, p. 1).

Tableau 3. Protection moyenne appliquée en 2004 (en \%)

\begin{tabular}{||l|l|l|l|l||}
\hline \hline & Monde & Pays Riches & PED & PMA \\
\hline Produits agricole : & 20,6 & 20,1 & 21,9 & 14,4 \\
primaire et semi-transformés & 14,9 & 14,8 & 15,3 & 9,8 \\
finaux & 24,3 & 23,4 & 26,6 & 17,1 \\
\hline Produits industriels : & 4,6 & 2,6 & 9,0 & 11,8 \\
primaire et semi-transformés & 3,1 & 1,5 & 6,5 & 9,5 \\
finaux & 5,9 & 3,6 & 11,7 & 13,9 \\
\hline Extraction et énergie : & 1,9 & 0,9 & 4,9 & 12,9 \\
primaire et semi-transformés & 1,4 & 0,6 & 3,8 & 13,6 \\
finaux & 3,8 & 1,9 & 7,5 & 12,1 \\
\hline Tous produits: & 5,3 & 3,5 & 9,6 & 12,3 \\
primaire et semi-transformés & 3,4 & 1,9 & 6,7 & 10,1 \\
finaux & 7,3 & 5,0 & 13,0 & 14,2 \\
\hline \hline
\end{tabular}




\subsection{Un système durable?}

29 La durabilité n'est pas ici entendue au sens traditionnel du développement durable: l'environnement n'est pas directement concerné, au contraire de la préoccupation à comprendre les conditions d'un développement futur exempt de crise économique structurelle (crise du système économique). À la façon de M. Chauchat et C. Perret (2006), par système durable, nous entendons un système économique permettant une croissance pérenne et une équité passant par une répartition des richesses et une fiscalité sûre et stable dans le temps.

L'explication de la durabilité de la situation (absence de faillite économique du territoire, malgré un taux de couverture très faible et une fiscalité protectionniste) réside uniquement dans les transferts métropolitains. En pratique, ces transferts font que la Nouvelle-Calédonie ne subit pas la contrainte extérieure. Les transferts de l'État sont supérieurs au déficit des opérations sur biens et services avec l'extérieur. Ces transferts équilibrent le déficit de la balance des transactions courantes (DME, 2005, p. 29). On peut noter que le niveau de transfert par habitant en Nouvelle-Calédonie est quarante fois supérieur à la moyenne des transferts par habitant des autres petits États insulaires du Pacifique (DME, 2005, p. 29).

31 Si les transferts sont la clé d'une situation durable, la question du maintien de ces transferts et de leur niveau doit être posée, a fortiori, dans le contexte d'émancipation que connaît la Nouvelle-Calédonie. La baisse la plus visible (et la plus brutale) des transferts pourrait être la diminution de l'indexation des salaires dont bénéficient les fonctionnaires $(+73 \%)$. La première conséquence économique d'une telle baisse serait une diminution du pouvoir d'achat moyen, avec donc difficultés pour les entreprises locales à écouler leurs productions (sauf à diminuer les marges). Cet effet pourra être renforcé par l'augmentation consécutive du nombre de faillites et donc de sans-emploi. Les entreprises pourraient difficilement retrouver une marge d'action via des baisses des prix, puisqu'elles ont justement besoin de la protection, du fait de leurs coûts de production élevés ; la Nouvelle-Calédonie se retrouverait alors contrainte par son système archaïque protectionniste.

«Cette situation, qui signifie perpétuation de la dépendance économique et donc de l'assistanat, peut-elle aller de pair avec le processus d'émancipation de la NouvelleCalédonie vis-à-vis de la métropole? Que se passera-t-il si les transferts en provenance de la métropole diminuent ou si l'indexation des fonctionnaires est réduite? Comment alors les productions calédoniennes pourront-elles être écoulées? Et si les prix sont diminués (via notamment l'abaissement des tarifs douaniers), ne seront-elles pas obligatoirement évincées par les productions étrangères? Faudrait-il dans ce cas généraliser les contingentements et diminuer les montants autorisés? » (Perret 2002).

\section{Les principes généraux de la TVA}

\subsection{Le mécanisme des déductions}

La France est le premier pays à avoir introduit la TVA (introduction le 10 avril 1954 et développement dans les années 1960). Les années 1990 ont été marquées par une augmentation spectaculaire du nombre de pays ayant adopté la TVA : la quasi-totalité des économies des ex-Pays de l'Est, beaucoup de pays en développement en Afrique sub- 
saharienne, en Asie et dans le Pacifique (où l'adoption de la TVA a bénéficié d'une impulsion du fait des répercussions de la libéralisation des échanges extérieurs ${ }^{12}$ ) et dans les petites économies insulaires, pour lesquelles la TVA était inconnue dix ans auparavant. En 2005, 136 pays avaient adopté la TVA (les États-Unis apparaissant comme une exception majeure). Cette taxe représente en moyenne un quart de l'ensemble des recettes fiscales. Les modalités d'application de la TVA à travers le monde sont très diverses : assiettes et activités auxquelles elle s'applique varient en fonction des pays.

La multiplicité des cas s'accompagne d'une diversité de visions de ce que doit être la taxe, mais plusieurs aspects fondamentaux font consensus. Premièrement, l'assiette finale de la taxe doit être la consommation. Une telle TVA suppose la déductibilité de la taxe payée sur les biens d'investissement, elle ne doit pas fausser les prix d'achat et de vente, et doit donc permettre d'assurer l'efficience économique (la taxe est neutre et n'influence pas les décisions des opérateurs). La TVA étant perçue à chaque stade de la production, pour s'assurer qu'elle ne pèse que sur la consommation, il faut également garantir la déductibilité complète de la taxe payée sur les inputs et éviter les ruptures dans la chaîne de la TVA, comme une exonération des inputs ${ }^{13}$. Il s'agit là du mécanisme des déductions : les assujettis doivent facturer la TVA sur toutes leurs ventes, mais peuvent également déduire la taxe qu'ils ont acquittée sur tous leurs achats.

L'effet cumulatif de la taxation, à chaque étape du cycle de production, est neutralisé par le droit à déduction. De sorte que l'agent économique, qui accomplit habituellement des opérations passibles de la TVA devient, pour chacune d'elles, et dans la même période, à la fois redevable envers le Trésor de la TVA facturée à son client et créancier du Trésor à raison de la taxe qu'il a payée à ses fournisseurs ${ }^{14}$.

Ce système a pour avantage de garantir les recettes dans la mesure où elles sont collectées tout au long du processus de production (contrairement à une taxe de vente au détail) mais sans fausser les décisions en matière de production, comme le ferait une taxe sur le chiffre d'affaires ${ }^{15}$. En pratique, il est procédé à un double calcul : on totalise d'un côté la taxe exigible liquidée en fonction des opérations imposables réalisées au cours de la période de référence; de l'autre on additionne la taxe dont le droit à déduction a pris naissance au cours de cette même période. On procède ensuite au calcul du solde consistant à soustraire la taxe récupérable de la taxe due. Lorsque la taxe exigible est supérieure à la taxe récupérable, le solde sera seul à acquitter; dans le cas où le solde est négatif, le crédit dégagé se reporte sur les opérations réalisées au cours de la période qui suit. Le graphique $\mathrm{n}^{\circ} 1$ illustre le mécanisme des déductions. 


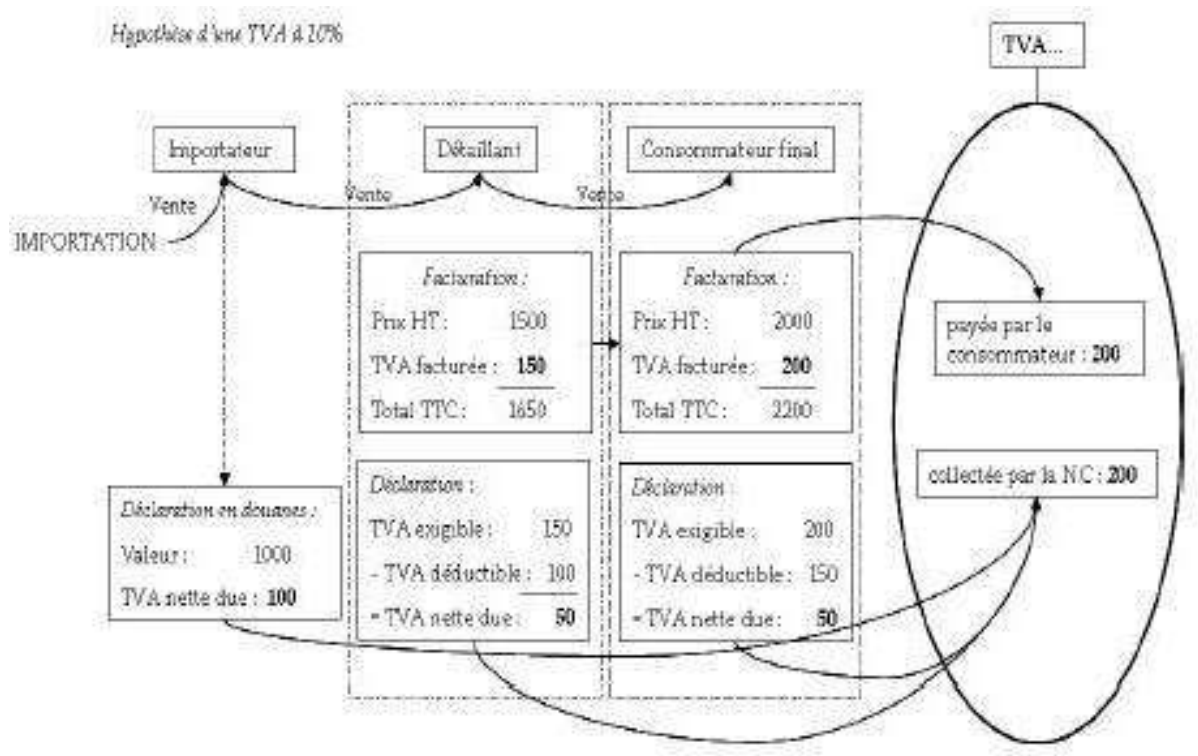

\subsection{Les avantages}

in

Le fait que la TVA n'ait été supprimée définitivement que par deux pays après son adoption illustre ses mérites : la Grenade (où la TVA a été introduite en 1986 et supprimée peu après) et Belize (introduite en 1996, la TVA a été supprimée en 1999). Concernant les risques de fraude, on peut noter que le mécanisme de déduction, qui repose sur le principe du crédit sur facture reliant explicitement le crédit de taxe sur les inputs de l'acheteur à la taxe acquittée par le fournisseur de ces inputs, présente l'avantage de décourager la sous-évaluation frauduleuse des ventes intermédiaires.

$\mathrm{Au}$ niveau des coûts d'administration, il est convenu qu'ils sont inversement proportionnels à la simplicité des mécanismes de la TVA ${ }^{16}$ (donc faibles avec un taux unique et un seuil d'assujettissement élevé).

Le seuil de chiffre d'affaires à partir duquel l'assujettissement à la TVA est obligatoire constitue un élément décisif dans la structure et la mise en œuvre d'une TVA ${ }^{17}$. Le niveau du seuil d'assujettissement à la TVA varie considérablement d'un pays à l'autre, allant de quelques milliers de dollars américains à plus de 200000 dollars américains selon les cas (environ 150000 euros, soit environ 18 millions CFP). Même au sein de l'UE, bien qu'un cadre juridique commun régisse la TVA dans les États membres, le seuil est très variable. L'attrait d'un seuil élevé tient à la constatation selon laquelle une large part des recettes de TVA potentielles provient généralement d'une proportion réduite d'entreprises. Un seuil élevé permet non seulement d'économiser des ressources administratives, mais également d'améliorer l'efficacité et le rendement de la TVA. Les autorités peuvent se permettre d'exonérer les petits entrepreneurs sans risquer de conséquences néfastes en termes de recettes, dès lors que ces petits entrepreneurs génèrent une valeur ajoutée limitée.

\subsection{Le cas des petits pays}

Existe-t-il des pays si petits que la TVA n'y serait pas appropriée? La question est importante pour l'avenir et l'extension de cet impôt, puisqu'un grand nombre de pays ne 
l'ayant pas encore adopté sont des petits pays. Comme indiqué précédemment, les recettes de TVA augmentent avec la part du commerce international dans l'économie. Les petits pays ayant tendance à être plus dépendants des échanges commerciaux (petites économies ouvertes), on peut a priori déduire que la TVA donne de meilleurs résultats dans ces pays que dans les grands. De plus, une taxe sur la consommation aura le plus souvent tendance à moins fausser l'économie qu'un régime de droits de douane pour un montant de recettes équivalent. La décision d'adopter ou non la TVA dépendra également, dans le cas d'un petit pays, de la différence de coûts de recouvrement entre la TVA et l'autre source potentielle de recettes.

Dans le cas où la TVA se substituerait entièrement à une recette tarifaire, et où la protection ne serait pas qu'un motif de recettes fiscales mais bien également une protection de marché (Nouvelle-Calédonie, notamment), la substitution de taxe signifierait un abaissement de la protection. Dans ce cas, un maintien de la protection passerait forcément par une politique de contingentements. Le problème tiendrait alors au fait que les quotas sont un instrument de politique commerciale problématique pour ce qui est du bien-être social. En termes purement économiques, le quota est un instrument moins efficace que le tarif; le système des prix ne peut plus jouer son rôle de signal qui permettrait une allocation efficiente des ressources. De plus, le bien-être des consommateurs est détérioré par la réduction de l'éventail de choix.

La question de la TVA peut être analysée au regard de l'expérience polynésienne. Dans cette COM, la TVA a été instaurée par étape à partir de $1998^{18}$, sans que cela ne crée de troubles et avec une conséquence plutôt désinflationniste. Pour autant, ce qui est bon pour la Polynésie peut-il l'être pour la Nouvelle-Calédonie?

\section{Les principes généraux de la TVA sociale}

\subsection{Les deux optiques de la «TVA sociale » (TVAS)}

Une TVA sociale signifie envisager un financement de la protection sociale (souvent chroniquement déficitaire) par la taxe. Le principe de la TVA sociale consiste à transférer le montant des charges sociales intégrées dans le prix de revient des entreprises, vers ce qui sera toujours une cotisation sociale mais enregistrée de manière comptable comme la TVA, c'est-à-dire en dehors du prix de revient. Le prix TTC des produits fabriqués localement restera lui inchangé, les entreprises répercutant dans les deux cas cette charge à leur client dans le prix de vente. Si le principe est clair, le concept de TVA sociale n'est pas pour autant uniforme. Les promoteurs de cette taxe sont nombreux, mais il n'existe pas à ce jour de cadre d'analyse de référence et en réalité le concept correspond à deux optiques différentes. La première optique se décline en deux versions.

Première version : la TVAS comme mode nouveau de calcul des cotisations sociales. Dans cette optique, la TVAS n'est pas (contrairement à ce que son appellation laisse supposer) un prélèvement nouveau, mais un mode de calcul nouveau des cotisations sociales (la TVA «normale» est alors appelée "TVA fiscale»). La TVAS serait alors payée par les entreprises directement aux organismes sociaux et entrerait dans l'assiette de calcul de la TVA fiscale. Le calcul et la perception de la TVAS sont semblables à ceux de la TVA : elle s'appliquerait à tous les biens vendus sur le territoire considéré et ne s'appliquerait pas aux exportations. Les prix à l'export sont alors «hors toutes taxes» et les prix sur le marché intérieur sont toutes TVA comprises. C'est en cela que réside l'intérêt du 
mécanisme de transfert des charges sociales sur une TVAS, qui se traduit par un double gain: diminution des prix à l'export par la suppression des cotisations (et donc accroissement de la compétitivité); augmentation du prix des produits importés par l'addition de la TVAS à la TVA (protection du marché intérieur). L'instauration d'un tel mécanisme ne modifierait pas les prix intérieurs (puisque le montant de TVAS ajouté au prix hors TVA est égal au montant des cotisations) et serait donc normalement neutre pour les consommateurs. Dans cette version, il y a donc explicitement deux TVA, une « sociale » et une « normale » (« fiscale »).

\section{Encadré 1.Exemple de TVAS}

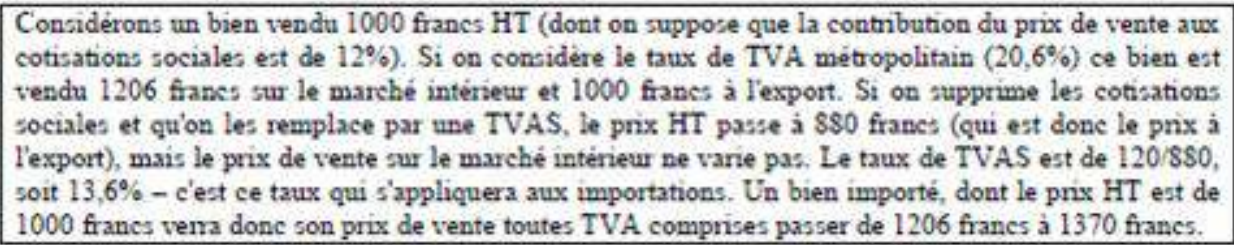

Deuxième version : la TVAS comme un élément de la TVA. Dans cette optique, il s'agit de financer une partie des dépenses de protection sociale non plus par des cotisations sociales assises sur les salaires, mais par une hausse des taux de la TVA. Les cotisations sont diminuées ou supprimées et les taux de TVA sont augmentés de façon à maintenir les recettes globales. Les budgets sociaux peuvent ensuite être alimentés par les recettes de la TVAS. Dans cette version, il n'y a donc qu'une seule TVA.

La deuxième optique est celle de la cotisation sociale sur la valeur ajoutée (CSVA). Dans ce cas de figure, l'allègement de charge est financé par une taxe qui pèse directement sur la valeur ajoutée ${ }^{19}$ et non, comme la TVA, sur la consommation des ménages. Les mécanismes économiques sont alors différents de ceux à l'œuvre dans une TVA ou TVAS. En effet, si on reporte le financement des cotisations sociales sur l'assiette de la TVA (TVAS), la réforme concerne le travail, mais le coût du capital n'est pas affecté par la hausse de la taxe. Mais si l'assiette de substitution est plus large, qu'elle coïncide avec la valeur ajoutée, c'est-à-dire le PIB, alors, en réponse à la baisse des cotisations sociales, la taxe est reportée à la fois sur le travail et le capital. En conséquence, il y a une baisse du coût relatif du travail. C'est cette variante de la TVA sociale qui a été vantée par le précédent Président de la République en janvier $2006^{20}$.

\subsection{Les gains attendus d'un mécanisme de TVAS}

De façon générale, les avantages peuvent être divisés en trois catégories. Premier avantage : la lutte contre les délocalisations, d'une part, en diminuant le coût du travail pour favoriser l'emploi ${ }^{21}$; d'autre part, en supprimant toute distorsion de nature fiscale dans le coût de production entre les biens, selon qu'ils sont ou non fabriqués dans le pays considéré. Cela signifie que la TVAS frapperait les biens domestiques comme les biens importés et serait donc non discriminatoire quel que soit le pays d'origine du produit. A contrario, les cotisations sociales peuvent être vues comme un impôt discriminatoire qui frappe les productions domestiques et exonère les productions étrangères. Les productions domestiques sont donc handicapées à l'export et les importations qui les concurrencent sur le marché domestique sont facilitées : en ce sens le système actuel peut être considéré comme "un droit de douanes à l'envers ». La baisse du coût du travail 
devrait stimuler l'emploi. Les simulations du Comité d'observation économique de la CCI de Paris laissent augurer, en prenant le scénario le plus crédible, une baisse du taux de chômage de 0,13 point après deux ans de fonctionnement d'une TVAS en France (Marini 2006, p. 2.). Ce n'est donc pas là l'avantage attendu en Nouvelle-Calédonie (au contraire de l'équilibre des comptes sociaux). Deuxième avantage : la réduction des déficits publics, par trois canaux. En premier lieu, en appliquant la TVAS aux retraites de fonctionnaires, celles-ci ne seraient donc plus financées par l'État. En second lieu, en taxant les produits importés ( $a$ priori de plus en plus abondants) on ferait participer les producteurs étrangers à la protection sociale du pays considéré. Actuellement une entreprise qui, pour fabriquer et vendre sa production sur le territoire calédonien, emploie dix salariés, doit payer dix cotisations sociales, tandis que celle qui importe de l'étranger les mêmes biens avec une deux employés seulement, n'en paye que deux. En troisième lieu, taxer la valeur ajoutée (deuxième optique) signifie une assiette plus large que les salaires; le montant collecté varierait donc moins que les recettes de la sécurité sociale selon les cycles économiques. Troisième avantage : la promotion du commerce extérieur grâce à la diminution du prix des biens à l'export (vendus hors TVA) ${ }^{22}$ et à l'augmentation du prix des biens importés ${ }^{23}$.

Pour synthétiser au mieux les avantages d'une TVAS, on peut dire qu'un tel dispositif permet d'améliorer la compétitivité sans réduire la demande globale, qui nourrit la croissance et l'emploi.

\subsection{Les inconvénients économiques attendus d'un mécanisme de TVAS}

Le prélèvement de TVA est de fait limité à la part de revenu consacrée à la consommation individuelle, cette part étant proportionnellement d'autant plus petite que le salaire est haut (plus le revenu est élevé et plus l'épargne est forte). Pour les bas salaires, le pourcentage de TVA est alors pris sur la totalité du salaire. En revanche, pour les hauts revenus, la TVA ne touche que la partie dépensée (c'est-à-dire à l'exclusion de l'épargne). Ainsi, le système de la cotisation sociale est progressif, alors que la TVA est dégressive. La TVA peut donc apparaître comme un impôt «antisocial ». Le problème d'équité devient crucial si on se concentre sur le cœur du dispositif (la substitution des cotisations par la TVAS), puisque la hausse des taux de TVA touche des individus qui ne sont pas directement concernés par les allègements de cotisation.

Concernant l'inflation, P. Marini estime que l'instauration d'une telle taxe présente un « risque inflationniste limité certes mais réel.» (Marini 2006, p. 5.) Le risque inflationniste de la TVAS est réel si la taxe se substitue aux charges patronales (et non salariales), et uniquement si les entreprises ne répercutent pas la baisse des charges patronales dans leur prix de vente (hausse de la marge - effet d'aubaine); alors, sous ces hypothèses, les consommateurs pourraient « supporter » la taxe sous la forme d'un choc inflationniste.

Dans un registre proche, certains estiment que les entreprises de transformation locale sont sources de réticence à la TVA du fait de sa transparence. Actuellement, les taxes en cascade font que le consommateur est incapable de connaitre la composition du prix du produit qu'il achète. Avec une TVA se substituant aux taxes, le système devient largement transparent, il ne serait donc pas simple de camoufler des marges très élevées. M. Chauchat (2006, p. 92) voit dans cette transparence des marges la principale cause de réticence de la production locale calédonienne vis-à-vis de l'instauration de la TVA. 
52 La deuxième optique de la TVAS (les cotisations sociales assises sur la VA) pose des questions particulières. Calculer les cotisations sociales sur la valeur ajoutée revient à remplacer le mode de calcul actuel où les cotisations sont fonction des salaires par un autre où les cotisations sociales seraient fonction de la valeur ajoutée de l'entreprise. Ce simple changement du mode de calcul des charges sociales n'allègerait pas le poids sur les entreprises des prélèvements sociaux qui pèseraient toujours sur les prix de revient et ne changerait pas non plus le prix des importations. Un tel mécanisme n'aurait donc aucun des deux principaux effets bénéfiques de la TVA sociale.

En outre, la valeur ajoutée n'est pas facile à mesurer dans certains secteurs comme la banque, l'assurance ou les services non marchands et il faudrait prévoir des dispositions spéciales pour eux. Le seul effet positif resterait alors un rééquilibrage des coûts des facteurs en faveur du travail (lutte contre les délocalisations).

Pour présenter la faisabilité d'une TVA sociale en Nouvelle-Calédonie, nous ferons donc référence aux deux premières optiques de la TVAS et pas aux cotisations sociales assises sur la valeur ajoutée.

\subsection{Autres problèmes}

\subsubsection{La question de la structure économique}

La TVA est une taxe reconnue comme « facile » à collecter. Ça ne signifie pas pour autant qu'elle soit optimale; d'autres taxes peuvent être plus rentables. Pour les petites économies ouvertes la question qui se pose est celle de la comparaison entre un droit de douane uniforme et une TVA - la taxe douanière est encore plus facile à collecter. L. Ebrill et al. (2002) estiment ainsi que pour une petite économie insulaire les écarts de coûts de gestion entre la TVA et les droits de douane « devraient être assez élevés pour l'emporter sur le facteur efficience d'une TVA. » Surtout, les auteurs rappellent que l'intérêt de la TVA dépend de la structure de l'économie (et surtout pour une économie insulaire). Moins la production intérieure comportera de stades de transformation et moins la TVA présentera d'avantages par rapport à un tarif douanier. À titre d'illustration, si on fait l'hypothèse de coûts de gestion nuls pour les deux types de fiscalité, et si on prend le cas (abstrait) d'un bien produit localement sans aucun stade de transformation, le droit de douanes et la taxe sont alors équivalents. Mais c'est bien parce que le droit de douane est plus facile à collecter que le nombre de stades de production devient un facteur discriminant. "Plus la part des importations dans le PIB et la proportion de la consommation importée sont faibles, plus la part des biens intermédiaires dans les importations est élevée, et plus grands sont les avantages d'une TVA, qui augmentent aussi à mesure qu'une économie se développe.» (Ebrill et al 2002, p. 46).

\subsubsection{Quid de la CSG ?}

Notons qu'en métropole les problèmes structurels de déficits de la protection sociale ont appelé une première solution en faisant payer tous les revenus: épargne, chômage, retraite via la CSG en 1990. La CSG est un impôt ${ }^{24}$ efficace : son assiette est très large (tous les revenus des ménages) et son taux faible (1,08\% initialement). Pourtant la CSG ne change rien au coût du travail en France ni donc son attractivité par rapport à d'autres pays ou à la mécanisation. La CSG n'aurait donc pas les effets dynamisants d'une TVSA sociale $^{25}$. On constate d'ailleurs que si la CSG sert à combler les déficits elle ne les résout 
pas : annoncée comme ne devant jamais dépasser $1 \%$ lors de son instauration en 1990 en métropole, la CSG correspond actuellement à un taux de 7,5\% des revenus d'activité ${ }^{6}$ : la taxe croît donc avec les déficits qu'elle combat.

\section{Quelles possibilités en Nouvelle-Calédonie?}

\subsection{La réforme avortée (2004-2006)}

57 La nécessité de réformer la fiscalité calédonienne semble peu contestée. La multiplicité des taxes en Calédonie, qui souvent s'appliquent en cascade, font de la fiscalité calédonienne un labyrinthe dans lequel il est souvent bien difficile de trouver son chemin : Taxe Générale à l'Importation (TGI), Taxe de Base à l'Importation (TBI), droits de douanes, Taxe sur le fret aérien, Taxe de péage, contribution des patentes, etc., et surtout multitude d'exonérations. Le premier objectif d'une réforme visera à simplifier ce système c'est-à-dire substituer une taxe unique à un ensemble de taxes. En ce sens, l'option de la TVA apparaît comme une des plus naturelles.

58 En outre, le remplacement d'une multitude de "petites " taxes par une "grande taxe » (la TVA) est favorable à la croissance économique («tax smoothing») et cette substitution doit également avoir un impact désinflationniste (Carbonnier 2006, p. 62).

Le gouvernement Thémereau (2004-2007) a initié une telle réforme de la fiscalité calédonienne. Ce projet prévoyait le remplacement de nombreuses taxes par une TVA. Le projet a cependant attisé les mécontentements des entrepreneurs et des commerçants locaux. Il n'a pu être mené à terme. On peut considérer que ce projet regroupait trois grandes familles d'objectifs : simplifier la fiscalité, la rendre plus dynamique, améliorer son efficacité.

60 Les deux premiers objectifs ont trait à la fiscalité douanière. Cette fiscalité est lourde et complexe, la gestion de la fiscalité se fait donc largement au détriment de son contrôle. Ainsi, les trois taxes budgétaires (TGI, TBI, TFA) comportent 8000 positions tarifaires. La TGI et la TBI comportent plus de 2600 cas d'exonérations. À quoi on peut ajouter que la TFA, affectée à l'Agence pour la Desserte Aérienne de la Nouvelle-Calédonie, pénalise le fret avion, alors qu'elle est destinée à financer la compagnie locale AIRCALIN. De plus, la fiscalité douanière a un impact néfaste sur les prix, elle alourdit le coût des biens d'équipement des entreprises. Comme elle est payée avant toute activité, elle représente une perte sèche quand la marchandise importée est ensuite perdue ou non entièrement exploitée. Enfin, les mesures d'exonération, visant justement à pallier ces inconvénients sont un coût de gestion supplémentaire pour les entreprises.

61 Le troisième objectif vise la suppression de deux impôts, la TSS et la patente. La TSS est une charge pour les entreprises, et peut être payée en cascade. De plus, affectée à la $\mathrm{CAFAT}^{27}$, elle ne constitue pas le bon instrument fiscal : elle est inéquitable et l'assiette est trop étroite. La patente apparaît comme un impôt anachronique (la taxe déterminée est fondée sur un tarif qui n'a jamais été actualisé); elle pèse sur les investissements des entreprises et les exportations et est en outre inéquitable, car très concentrée (cinquante patentés paient près de $50 \%$ de l'impôt) et essentiellement payée par le secteur du commerce.

62 Dans les projets du Gouvernement de la Nouvelle-Calédonie pour la TVA, la première étape devait comprendre ${ }^{28}$ la série de mesures suivante : suppression de plusieurs taxes 
existantes (TSS, TBI, TFA, Taxe de péage) ; diminution de la TGI sur un certain nombre de produits ; instauration d'une TVA à $6 \%$ (la TVA à $6 \%$ était supposée bénéficier d'une assiette de 370 milliards et donc générer une recette fiscale de 22,2 milliards CFP). La seconde étape devait comprendre la suppression totale de la TGI, la fixation du taux définitif de la TVA aux alentours de $10 \%$, l'ajustement des taxes de protection de la production locale. Deux taux de TVA étaient a priori prévus : un taux normal sur les biens et services; un taux réduit sur certains produits de première nécessité, sur les livres et journaux (produits de la culture), les équipements pour personnes handicapées et les médicaments. Des exonérations de TVA étaient également prévues pour les biens suivants : soins médicaux, enseignement, locations d'immeubles, opérations bancaires et d'assurance, carburants (essence et gazole), organismes sans but lucratif, pêche professionnelle (option facultative), exportations (avec récupération de la taxe sur les achats). Seules seraient obligatoirement assujetties à la TVA les entreprises réalisant un chiffre d'affaire annuel d'au moins 7,5 millions CFP. Les autres entreprises ne paieraient pas de TVA et ne pourraient donc pas non plus effectuer de déductions. Elles pourraient, si elles le souhaitaient, bénéficier de la réforme (pour pouvoir faire des déductions de TVA).

Ont été présentés les caractéristiques du système traditionnel de TVA et le projet avorté d'instauration de la TVA en Nouvelle-Calédonie. Cette instauration permettrait de moderniser la fiscalité calédonienne. Toutefois, eu égard à l'archaïsme de la fiscalité calédonienne, on peut considérer que le projet de réforme part d'une page blanche. Alors que ce débat est amorcé, notamment en métropole ${ }^{29}$, la question se pose de la mise en place d'une TVA calédonienne incluant le financement des régimes sociaux. C'est la problématique de la TVA sociale, vraisemblablement adaptée à la situation de la Nouvelle-Calédonie et au déficit de ses comptes sociaux.

\subsection{Une TVA sociale en Nouvelle-Calédonie}

Le Danemark est un des rares pays à avoir instauré un mécanisme de TVAS. Ce pays a en effet réalisé en 1987 une réforme fiscale qui s'est traduite par la suppression, entre 1987 et 1989 , des cotisations sociales versées par les employeurs. Le manque à gagner pour les finances publiques a été compensé par une hausse de la TVA de 22 à $25 \%$. En France, la loi Perben de 1994 a instauré un mécanisme de ce type à la Réunion, à la Guadeloupe et à la Martinique. Enfin, plus récemment, l'Allemagne a également introduit une TVAS au $1^{\mathrm{er}}$ janvier 2007 (voir Besson 2007, pour une présentation de l'introduction de la TVAS au Danemark et en Allemagne).

\subsubsection{La structure des cotisations sociales et des taxes en Nouvelle-Calédonie}




\begin{tabular}{|c|c|c|c|c|}
\hline RISQUE & EMPLOYEUR & SALARIE & TOTAL & $\begin{array}{l}\text { ASSIETTE } \\
\text { MENSUELLE (*) }\end{array}$ \\
\hline Maladie & $11,30 \%$ & $3,85 \%$ & $15,15 \%$ & 0 à 399100 \\
\hline Retraite & $9,10 \%$ & $3,90 \%$ & $13,00 \%$ & 0 ล 306500 \\
\hline Prestations familiales & $6,14 \%$ & - & $6,14 \%$ & $0 \div 306500$ \\
\hline Chômage & $1,52 \%$ & $0,34 \%$ & $1,86 \%$ & 0 ล 306500 \\
\hline Accidents du travail & 0,72 a $6,48 \%$ & - & 0,72 a $6,48 \%$ & 0 a 306500 \\
\hline Fonds social de l'habitat & $2 \%$ & - & $2 \%$ & 0 ล 258800 \\
\hline Formation professionnelle & $0,25 \%$ & - & $0,25 \%$ & $0 \div 306.500$ \\
\hline $\begin{array}{l}\text { Total des cotisations } \\
\text { rersées a la CAFAT }\end{array}$ & $\begin{array}{r}30,31 \% \\
(\text { hors AT) }\end{array}$ & $8,09 \%$ & $\begin{array}{r}38,40 \% \\
\text { (hors AT) }\end{array}$ & \\
\hline $\begin{array}{l}\text { Formation professionnelle } \\
\text { continue }\end{array}$ & $0,70 \%$ & - & $0,70 \%$ & Totalité du salaire \\
\hline $\begin{array}{l}\text { Contribution exceptionnelle } \\
\text { solidarité }\end{array}$ & - & $0,75 \%$ & $0,75 \%$ & $\begin{array}{l}\text { Sur partie du salaire } \\
\text { supéneure à } 306500\end{array}$ \\
\hline
\end{tabular}

Source : Centre des Liaisons Européennes et Internationales de Sécurité Sociale (CLEISS) : www.cleiss.fr. (*) Unité : F. CFP.

Le gouvernement de la Nouvelle-Calédonie envisageait d'affecter les recettes de la TVA de la façon suivante ${ }^{30}$. Le montant de la recette actuelle (estimée pour 2006) de la TFA devait servir à financer l'ADANC (sortie de défiscalisation des airbus locaux). La somme de 2 milliards de centimes additionnels à la patente devait alimenter un «fonds patentes». $80 \%$ du montant de la taxe de péage devait servir à financer le port autonome. Le montant de la TSS devait alimenter la CAFAT (à hauteur de 9,75 milliards ${ }^{31}$ ). Le reste (pour un total de 37 milliards) alimenterait le budget de la Nouvelle-Calédonie. Le graphique $\mathrm{n}$ $\circ 2$ représente cette répartition.

Graphique 2. Répartition prévue des recettes de TVA

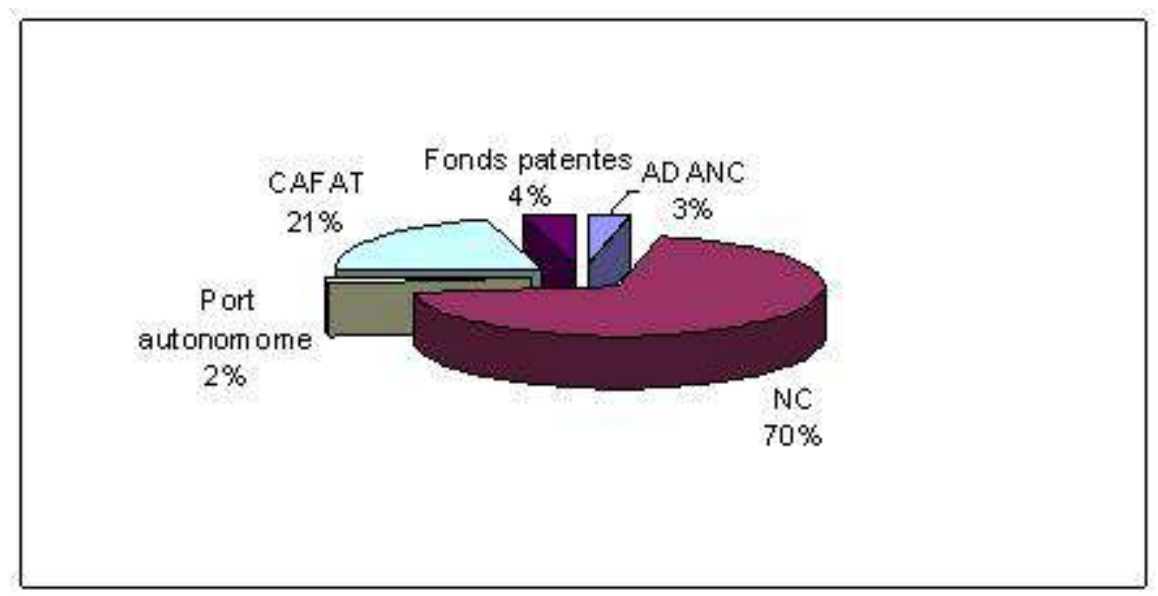

Source : Cabinet de D. Leroux membre du Gouvernement de la Nouvelle-Calédonie de 2004 à 2006 (en charge notamment de la fiscalité).

Bien que la CAFAT soit prévue comme destinataire des recettes de la TVA, il ne se serait pas agi pour autant d'une TVAS dans la mesure où la part de l'impôt dédié à la CAFAT est inchangée (même si cette part pourrait évoluer dans le temps et augmenter au fil des années). 
67 En réalité, le projet de TVA, plutôt que de combler le déficit de la CAFAT, était un projet à "déficit constant». Ce déficit est chronique ${ }^{32}$, même s'il varie selon les années et l'activité économique. En 2005, le déficit de la CAFAT était d'environ un milliard de F. CFP, pour un total de 54,3 milliards de cotisations. Les résultats se répartissaient ainsi : accidents du travail (déficit 183 millions), chômage (excédent de 497 millions), prestations familiales (excédent de 365 millions), RUAMM (déficit de 1,92 milliard), retraite (excédent de 197 millions) ${ }^{33}$. Il était encore moins question d'affecter une part du produit de la TVA au financement des retraites, alors que là aussi les déficits sont considérables $^{34}$, surtout pour une période de forte expansion économique.

\subsubsection{Pertinence}

Raisonner à déficits sociaux constants priverait donc le projet d'instauration d'une TVA en Nouvelle-Calédonie d'un aspect puissamment novateur (rappelons que l'ancien Président de la République française J. Chirac a prôné la TVA sociale depuis $2006^{35}$ ). En effet, il s'agissait essentiellement (i) de lutter contre le chômage et les délocalisations en diminuant le cout du travail, (ii) de restaurer l'équilibre des régimes sociaux. Le premier point n'est pas forcément pertinent en Nouvelle-Calédonie (chômage faible et en baisse, délocalisations a priori inexistantes), mais le second en revanche aurait toute son importance. Surtout, limiter l'objectif de l'aspect « social» de la TVA à une cible unique (les déficits sociaux) permettrait, à la fois sur le fond et en terme d'affichage, d'éviter l'aspect TVA «fourre-tout ». C'est en ce sens que l'instauration d'une TVA sociale en Nouvelle-Calédonie serait réellement une réforme moderne et novatrice. En l'absence d'un réel arrimage social, on se retrouve avec une TVA « normale », c'est-à-dire avec des avantages connus à long terme, mais aussi des incertitudes liées au cadre de petite économie insulaire et les possibles inconvénients inflationnistes de court terme.

Pour ce qui est de la structure économique, on conçoit bien qu'une TVA normale n'a pas de raison évidente ou définitive de l'emporter sur une taxe douanière bien calibrée ${ }^{36}$. C'est pour cela qu'une réforme telle que la TVA sociale se justifie avec les avantages mentionnés.

\subsubsection{Illustration}

Concernant les simulations relatives à un passage à la TVAS, la tâche s'avère complexe. ${ }^{37}$ Afin de faciliter la compréhension du mécanisme envisagé, nous nous limiterons donc ici à l'illustrer par un exemple, dans le cas de produits fabriqués en Nouvelle-Calédonie.

Considérons ainsi le cas d'une industrie important des inputs (matières premières) taxés par les douanes à $33 \%$. On suppose que les matières premières coûtent 30 , les amortissements 20 et les salaires hors cotisations patronales 32 . Le montant des taxes douanières s'élève donc à 10 (c'est-à-dire 30x33) et les cotisations patronales à 10 également (arrondi 32x30,3\%). L'exemple détaille la situation actuelle; la situation d'une TVA se substituant parfaitement aux taxes douanières (c'est-à-dire à recettes constantes) ; enfin la situation d'une TVA se substituant aux taxes douanières et d'une TVAS se substituant aux cotisations patronales (recettes constantes). Dans le troisième cas, on considère donc l'hypothèse selon laquelle la totalité des charges patronales est transférée sur la valeur ajoutée, sans que rien ne soit changé quant aux parts salariales (retenues sur salaires), étant entendu que d'autres combinaisons et d'autres taux sont possibles. 
Tableau 5. Exemples d'une TVA et d'une TVAS en Nouvelle-Calédonie

\begin{tabular}{||l|c|c|c||}
\hline & $\begin{array}{c}\text { Situation } \\
\text { actuelle }\end{array}$ & $\begin{array}{c}\text { Situation avec } \\
\text { TVA }\end{array}$ & $\begin{array}{c}\text { Situation avec } \\
\text { TVAS }\end{array}$ \\
\cline { 2 - 4 } & 30 & 30 & 30 \\
\hline Matières premières HT & 10 & 0 & 0 \\
\hline Taxes douanières & 20 & 20 & 20 \\
\hline Amortissements équipements & 32 & 32 & 32 \\
\hline Salaires hors cotisations pat. & 10 & 10 & 0 \\
\hline Cot pat. versées à la CAFAT & 8 & 8 & 8 \\
\hline Profits avant impót & 110 & 100 & 90 \\
\hline Prix de vente HT & non pertinent & 100 & 90 \\
\hline Prix de vente hors TVA & non pertinent & non pertinent & 90 \\
\hline Prix de vente hors TVAS & 0 & 10 & 10 \\
\hline TVAS versée & 0 & 0 & 10 \\
\hline TVA versée à la CAFAT & 110 & 110 & 110 \\
\hline \multicolumn{2}{|l|}{} & & \\
\hline Prix de vente TTC & \multicolumn{3}{|c||}{} \\
\hline
\end{tabular}

Dans cet exemple, (Descombels 2007, p. 146) qui vise à expliquer les mécanismes de TVA, le taux de TVA serait de $10 \%$ et le taux de TVAS serait de $22 \%(20 / 90)$.

3 On montre que le prix de vente au consommateur calédonien serait inchangé, pour des taux de TVA et TVAS correctement calibrés, l'économie dans son ensemble bénéficiant des avantages de la TVAS (ou simplement de la TVA) : souplesse de trésorerie pour les entreprises, transparence des prix, fin du « droit de douane à l'envers » (TVAS), meilleure compétitivité, dynamisation de l'économie, etc. Au niveau des produits importés, leurs prix seront majorés de la TVA ou TVAS, c'est-à-dire dans ce dernier cas de $22 \%(20 / 90)$.

Bien que l'exemple du tableau 5 ait un rôle pédagogique et ne soit pas prospectif, ce taux de $22 \%$ est vraisemblablement proche de celui qui devrait prévaloir dans l'hypothèse d'une prise en charge de l'ensemble des dépenses de la CAFAT par la TVAS. En effet, le Gouvernement de la Nouvelle-Calédonie envisageait de remplacer 36 milliards de recettes fiscales par un taux de TVA d'environ $8 \%$. Les cotisations CAFAT se montent à environ 54 milliards (plus un milliard de déficit, plus le déficit de la CLR). On arrive donc à environ 92 milliards de F. CFP, qui correspondraient alors globalement à un taux de TVA globale (TVA «fiscale» plus TVA sociale) de $20,5 \%^{38}$. L'effet sur les prix serait globalement neutre ${ }^{39}$ puisque la pression fiscale de la TVA globale se substituerait à celle frappant les importations et aux cotisations patronales.

5 Si certains biens nécessitaient transitoirement des protections supplémentaires, la TCPPL pourrait alors intervenir.

\section{Conclusion}

La réforme fiscale visant à instaurer une TVA, portée de 2004 à 2006 par le gouvernement de la Nouvelle-Calédonie, est restée au stade de projet. Clairement, ce projet a coalisé les mécontentements; l'industrie de transformation locale, notamment, s'y est déclarée hostile. Dès lors, il était inutile de vouloir imposer un impôt quand ceux qui doivent être chargés de sa collecte (les chefs d'entreprises, les commerçants) s'opposent à son instauration. 

contexte calédonien de cycles courts de transformation. Concernant l'industrie de transformation locale, la crainte a été celle d'une baisse de la protection via la TVA, car non compensée, notamment, par une hausse de la TCPPL. Ainsi, les entreprises calédoniennes risquaient d'être concurrencées sur leur marché par des importations à moindre coût. De telles situations pourraient en effet se produire et devraient alors être gérées au cas par cas. La baisse de la protection étant bénéfique pour les consommateurs (baisse des prix et hausse du pouvoir d'achat), il faut pouvoir articuler réforme fiscale, baisse de la protection et maintien de marges positives pour les entreprises. de protection ferait plus que doubler par rapport au projet avorté - les inquiétudes et réticences des industriels devraient donc nettement diminuer. Cela signifie que pour les industriels locaux, la situation s'améliorerait par rapport à la situation d'une TVA simple, puisque les importations concurrençant la production locale seraient plus fortement taxées à l'entrée.

Plus globalement, en admettant qu'une baisse de la protection demeure, la question à résoudre serait: comment conserver des marges acceptables avec des baisses de prix ? C'est-à-dire comment obtenir des baisses des coûts de production ? Cette baisse des coûts doit passer par une baisse des coûts de l'investissement; ici pourrait intervenir l'outil de la défiscalisation. Une utilisation de la défiscalisation au service du développement calédonien signifierait une orientation vers des projets industriels permettant d'améliorer la compétitivité de ces industries. C'est le modèle en vigueur dans les DOM, qui bénéficient de la Loi Girardin et des aides de l'Europe permettant l'absorption des coûts d'investissement, jusqu'à hauteur de $80 \%$. En Nouvelle-Calédonie, via la double défiscalisation, une prise en charge de $70 \%$ du montant des investissements industriels ciblés peut être atteinte.

Toutefois la défiscalisation est une dépense publique. À cet égard, la défiscalisation qui serait orientée vers l'investissement productif devrait globalement être soustraite à d'autres secteurs (la structure globale des investissements défiscalisés devrait donc être modifiée). On pourrait également objecter que la défiscalisation est une aide aux nouveaux projets, et qu'elle ne serait donc pas pertinente pour les entreprises existantes. En réalité, quantité d'entreprises calédoniennes s'appuient sur un appareil productif vieillissant. C'est le renouvellement de ces équipements, pour une meilleure productivité et des coûts moindres, qui pourrait bénéficier de la politique de défiscalisation. Ainsi, le champ d'ajustement par la TCPPL serait réduit et le raisonnement au cas par cas facilité.

81

De façon générale, l'aide devrait passer par une transparence de la part des entreprises en bénéficiant: pas (ou plus) d'aides sans contrepartie. En bref : la puissance publique devra s'assurer qu'une entreprise bénéficiant d'aides ne maintient pas des marges trop élevées (ce qui signifie que les aides profitent indirectement aux consommateurs). A contrario, une entreprise ne sollicitant pas d'aides de la puissance publique serait entièrement libre de ses marges. Ainsi, le passage à la TVA ou TVAS pourrait s'accompagner de nouvelles aides offertes aux entreprises, dont le corollaire serait aussi une politique de modération des prix au consommateur. 


\section{Annexe 1. Les différents taux de TVA dans le monde}

\begin{tabular}{|c|c|c|c|c|c|}
\hline Pays & 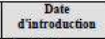 & \begin{tabular}{|c|} 
Taur \\
ordinaire (9)
\end{tabular} & Pays & $\begin{array}{l}\text { Date } \\
\text { dintroduction }\end{array}$ & $\begin{array}{l}\text { Taur } \\
\text { ordinaire }(90)\end{array}$ \\
\hline Af. du Sud & Sep. 1991 & 14 & $\mathrm{Nz}$ & Imisis6 & 12.5 \\
\hline Antilles neerl. & $\operatorname{mars}-99$ & 5 & Ougznds & Jull 1996 & 17 \\
\hline Cis. et Gaza & Jull 1976 & 17 & Ouzbekistan & $\operatorname{Jan} 1992$ & \\
\hline Corée & Jull 1977 & 10 & Palistan & Nov. 1990 & 15 \\
\hline Espagne & $\operatorname{Jan} 1986$ & 16 & Panama & $\operatorname{mans}-77$ & 5 \\
\hline Ghens & Déc. 1998 & 12.5 & Pappousie-NG & Juil 1999 & 10 \\
\hline Grice & $\operatorname{Ian} 1987$ & 18 & Paraguay & Jull 1993 & 10 \\
\hline Guatemsila & aouit-83 & 12 & Pays-Bas & $\operatorname{Tan} 1969$ & 19 \\
\hline Guinee & Jull 1996 & 18 & Perou & $\operatorname{Jan} 1973$ & 19 \\
\hline Haiti & Nov. 1982 & 10 & Philippines & $\operatorname{Jan} 1988$ & 10 \\
\hline Honduras & $\operatorname{Jan} 1976$ & 12 & Pologne & Jull 1993 & 22 \\
\hline Hongrie & $\operatorname{Jan} 1998$ & 25 & Portugal & $\operatorname{Jan} 1986$ & 19 \\
\hline Indoonesie & Ave 1985 & 10 & Rép. kirghize & $\operatorname{Jan} 1992$ & 20 \\
\hline Irlande & Nov. 1972 & 21 & Repp. slovague & $\operatorname{Tan} 1993$ & 19 \\
\hline Islande & $\operatorname{Jan} 19900$ & 24.5 & Rwanda & $\operatorname{Jan} 2001$ & 18 \\
\hline Israel & Jull 1976 & 17 & Roummie & Jull 1993 & 19 \\
\hline Indie & $\operatorname{Jan} 1973$ & 20 & Samoa & $\operatorname{Jan} 1994$ & 10 \\
\hline Jamaigute & Oct 1991 & 15 & Royame-Vni & Avr. 1973 & 17,5 \\
\hline Japon & Avr. 1989 & 5 & Russie & $\operatorname{Jan} 1992$ & 18 \\
\hline Jordanie & $\operatorname{Jan} 2001$ & 16 & Sénegal & mars -80 & 17 \\
\hline Kazakhstan & $\operatorname{San} 1992$ & 15 & Serbie-Slontenegro & Serbie 2005 . & 18 \\
\hline Keysa & $\operatorname{San} 1990$ & 16 & Singppour & Avr. 1994 & 5 \\
\hline Lesootho & Jull. 2003 & 14 & Slovenenie & Jull 1999 & 20 \\
\hline Lenorie & $\operatorname{Tan} 1992$ & 18 & Soudan & $\operatorname{Sin} 2000$ & 10 \\
\hline Liban & Fevv. 2002 & 10 & Sri Lanka & Avr. 1998 & 15 \\
\hline Lituanie & $\operatorname{Jan} 1992$ & 18 & Suede & $\operatorname{Jan} 1969$ & 25 \\
\hline Luxembourg & $\operatorname{Tan} 1970$ & 15 & Suisse & $\operatorname{Jan} 1905$ & 7.6 \\
\hline Maceidione & AvT. 2000 & 18 & Surinam & Avr. 1999 & 10 \\
\hline Madagascar & Sep. 1994 & 20 & Tadjkistan & $\operatorname{Jan} 1992$ & 20 \\
\hline Malani & $\max -89$ & 17.5 & Tainan & Avr. 1986 & 5 \\
\hline Mali & $\operatorname{San} 1991$ & 18 & Tanzanie & Jull 1998 & 20 \\
\hline Malte & $\operatorname{Jan} 1995$ & 18 & Thasilande & $\operatorname{Jan} 1992$ & 7 \\
\hline Maroc & Aṽ 1986 & 20 & Togo & Juil 1995 & 18 \\
\hline Marice & Sep. 1998 & 15 & Trinidad $\&$ Tob. & $\operatorname{Jan} 1990$ & 15 \\
\hline Manritanie & $\operatorname{Jan} 1995$ & 14 & Tunisie & Jull 1988 & 18 \\
\hline Mesigue & $\operatorname{Tan} 1980$ & 15 & Turkmenistan & $\operatorname{Jan} 1992$ & 20 \\
\hline Moidova & $\operatorname{Tan} 1992$ & 20 & Turquie & $\operatorname{Jan} 1985$ & 18 \\
\hline Mongoile & Full 1998 & 15 & Unuguay & $\operatorname{Jan} 1968$ & 23 \\
\hline Mozambigue & Juin 1999 & 17 & Ulsaine & $\operatorname{Jan} 1992$ & 18 \\
\hline Nambibie & Nov. 2000 & 15 & Vamuaru & aôt-98 & 12,5 \\
\hline Neepal & Nov. 1997 & 10 & Venezueia & OCt 1993 & 16 \\
\hline Nicanagua & $\operatorname{Janv} .1975$ & 14 & Venezulia & OCt 1993 & 16 \\
\hline Niger & $\operatorname{Jan} 1986$ & 19 & Viemam & $\operatorname{Jan} 1999$ & 10 \\
\hline Nigeria & $\operatorname{Jan} 1994$ & 5 & Zambie & Jull 1995 & 17,5 \\
\hline Norvige & $\operatorname{San} 1970$ & 24 & Zimbabre & $\operatorname{Jan} 2004$ & 15 \\
\hline
\end{tabular}

(Banque mondiale, FMI, OCDE, 2005)

\section{Annexe 2. Les échanges extérieurs et le taux de couverture de la Nouvelle-Calédonie}

Graphique 1A. L'évolution du commerce extérieur depuis 1998

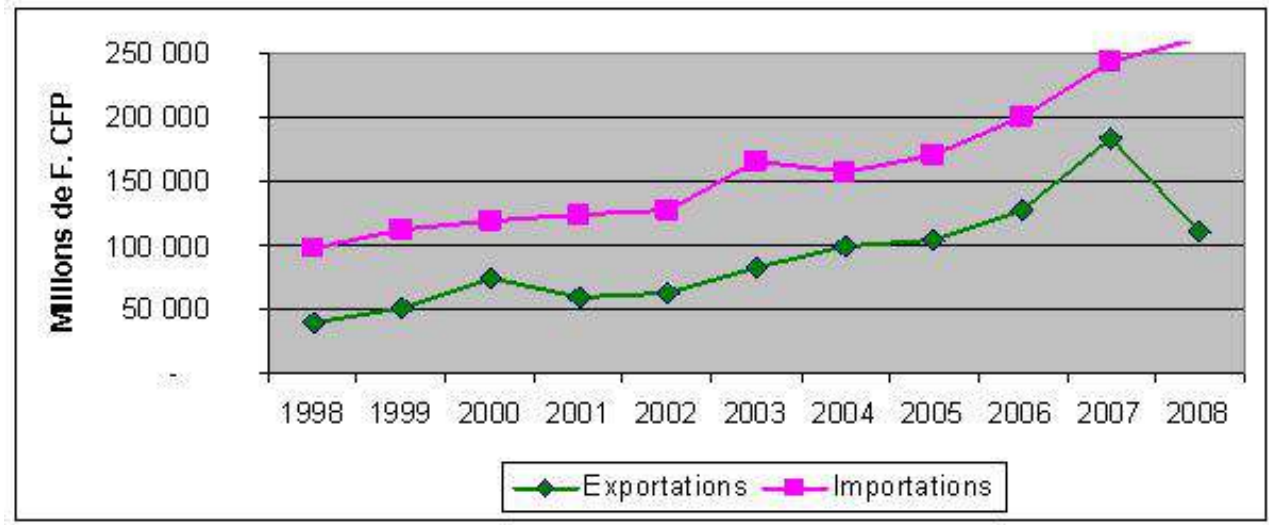




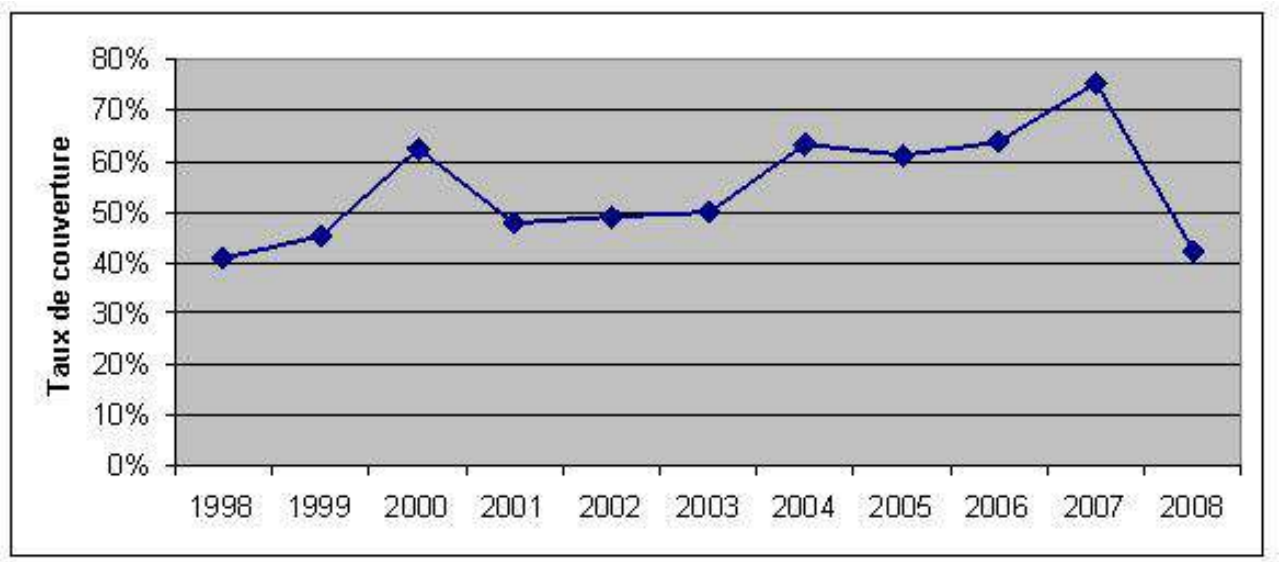

\section{Annexe 3. Les effets comparés d'un tarif et d'un quota}

Il est fréquent de considérer les quotas et les tarifs comme des instruments à peu près équivalents (dans le sens où tel quota correspond à un équivalent tarif qui permettrait d'importer les mêmes quantités). Cette hypothèse est cependant simplificatrice. Afin de comparer les effets des deux instruments nous reprenons l'analyse de J. Cassing et A L. Hillman (1985). L'action du gouvernement en matière de restrictions commerciales a deux principaux effets: une variation du profit des industries concernées, et une variation du prix de leurs produits. Considérons le schéma ci-dessous (Cassing et Hillman 1985, p. 282), qui représente une industrie.

Schéma 3A. Comparaison des effets du tarif et du quota

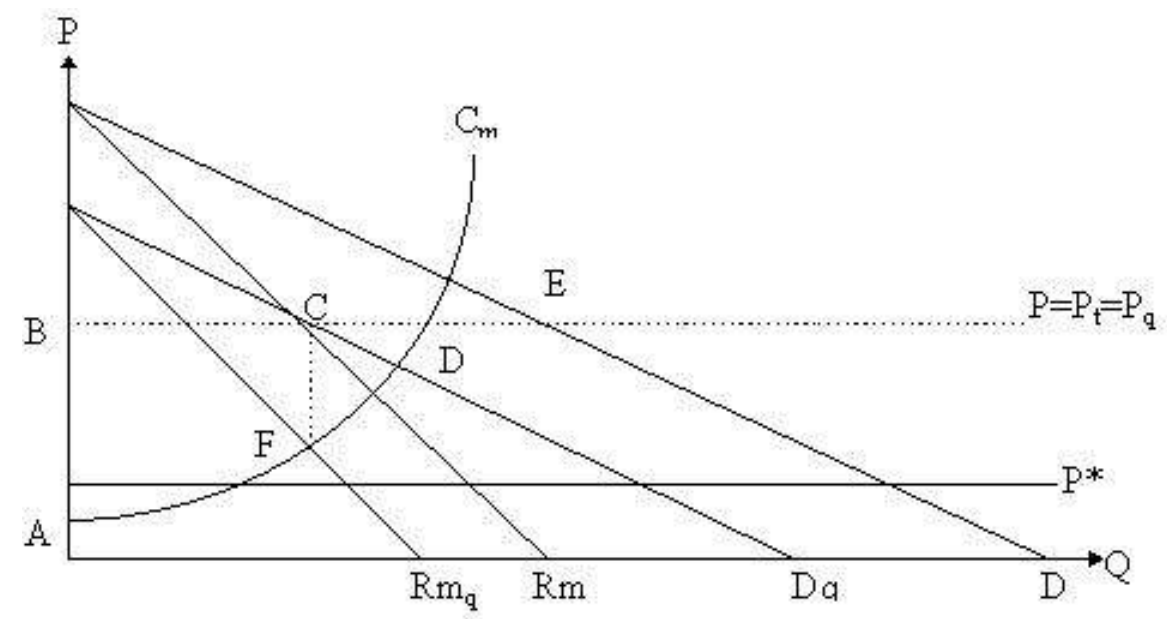

$\mathrm{P}^{*}$ est le prix mondial. Pt est le prix domestique, c'est-à-dire le prix mondial plus le tarif imposé sur les importations. D est la demande domestique. Rm est la recette marginale (on suppose une firme monopolistique). Dq est la demande résiduelle, c'est-à-dire la demande si on soustrait les importations. Rmq est la recette marginale correspondante. 

une faible inflation en tendance : l'indice des prix à la consommation a enregistré une variation moyenne annuelle de $1 \%$ entre 1995 et 2004. L'inflation est donc globalement modérée sur cette période avec des pics observés de 1999 à 2002 (essentiellement en 2002), où la réforme fiscale de la TVA a été mise en œuvre.

Après une période d'inflation d'amplitude plus soutenue $(+1,7 \% 0$ en $2000,+0,9 \%$ en 2001 et $+\%$ en 2002), les $0,8 \%$ de hausse observée entre décembre 2004 et 2003 et les 0,7\% entre 2003 et 2002 constituent une quasi stabilité. Le graphique 4A représente l'évolution de l'indice des prix en Polynésie française de 1995 à 2004. 


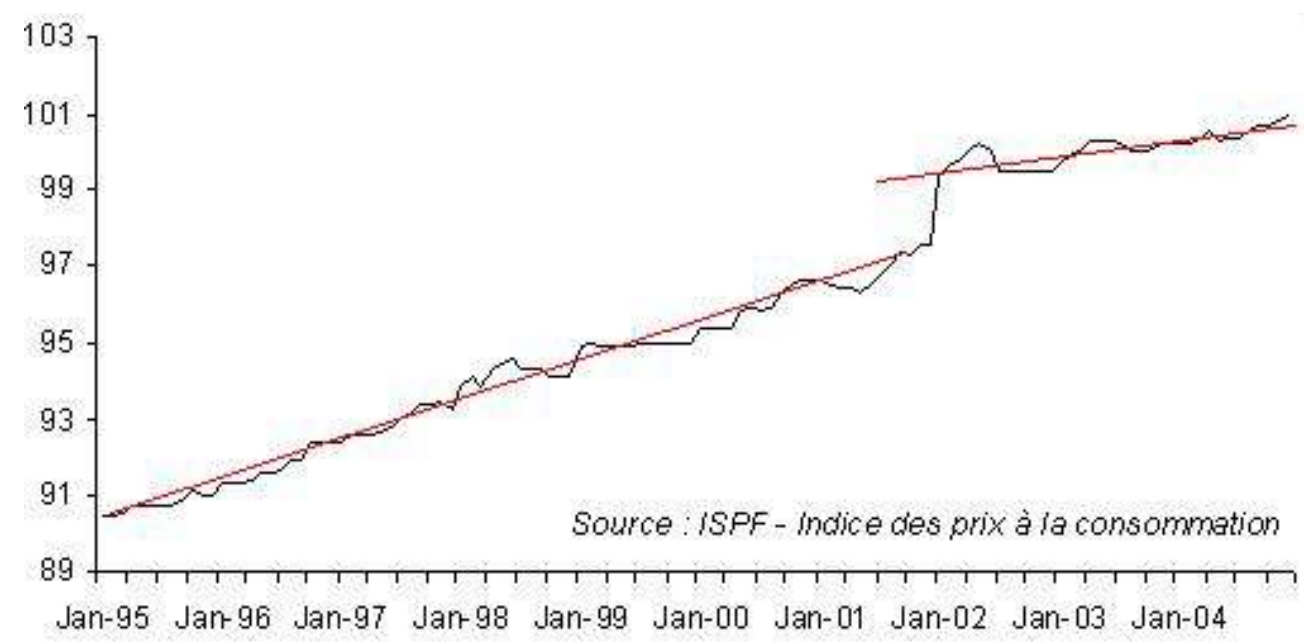

Descombels, 2007, p. 196

90 On constate donc bien un pic inflationniste en 2002. Le choc inflationniste (modéré) de 2002 a été aggravé par le phénomène suivant. Pendant la période de réforme fiscale, la baisse des droits d'entrée a été compensée par la hausse de la TVA. Mais en 2002, à la demande des industriels, le gouvernement polynésien a instauré une nouvelle taxe, la « taxe pour le développement local ». Cette taxe a en fait consisté à réintroduire les droits d'entrée sous un autre nom, pour les produits fabriqués importés concurrents de produits locaux. De ce fait, le protectionnisme a été maintenu (et même aggravé pour certains produits).

91 On constate également que les tendances inflationnistes (en rouge) se modifient après le pic inflationniste de 2002. Après la hausse de 2002, l'inflation se ralentit. Cela signifie que le choc est plus qu'absorbé : la poursuite des tendances montre qu'à partir de 2005 ou début 2006, le niveau des prix est inférieur à celui qui prévaudrait en absence de réforme fiscale. Cela signifie que l'instauration de la TVA induit un changement structurel désinflationniste dans l'économie polynésienne.

\section{BIBLIOGRAPHIE}

Atkinson A. B., Stiglitz J. E., 1976, "The design of tax structure: direct versus indirect taxation", Journal of Public Economics, 6, 55-75.

Avice F., Cannard P., 2007, "Rapport sur la TVA non perçue récupérable", Inspection générale des Finances, $\mathrm{n}^{\circ}$ 2007-M-016-02, 28 pages.

BANQUE MONDIALE, FMI, OCDE, La taxe sur la valeur ajoutée - Expériences et enjeux, Conférence sur la TVA du Dialogue Fiscal International, Rome, document de travail, 15-16 mars 2005, 33 pages. 
Barro R. J., 1979, "On the determination of public debt", Journal of Political Economy, vol. 87, 940-971.

Besson E., 2007, TVA sociale, Secrétariat d'État chargé de la prospective et de l'évaluation des politiques publiques, septembre, 157 pages.

Bohn H., 1990, "Tax smoothing with financial instruments", American Economic Review, vol. 80, $\mathrm{n}^{\circ} 5,1217-1230$.

Carbonnier C., 2006, "Fiscalité optimale et incidences fiscales analyses théoriques et estimations sur réformes françaises de la Taxe sur la Valeur Ajoutée et de la Taxe professionnelle, 1987-2004, Thèse de doctorat, EHESS, 225 pages.

Cassing J., Hillman A. L., 1985, "Political Influence Motives and the Choice Between Tariffs and Quotas", Journal of International Economics, 19, 279-290.

CEROM, 2005, La Nouvelle-Calédonie en mouvement, 2005, 47 pages.

Chambas G., 2005, "Afrique au Sud du Sahara, quelle stratégie de transition fiscale ?", Afrique contemporaine, $\mathrm{n}^{\circ} 213,133-163$.

Chauchat M., 2006, Vers un développement citoyen - Perspectives d'émancipation pour la Nouvelle-Calédonie, PUG, 229 pages.

Chauchat M., Perret C., 2006, "Désindexer pour un développement durable", in Chauchat M. 2006, Vers un développement citoyen - Perspectives d'émancipation pour la Nouvelle-Calédonie, PUG, 137-200.

Cremer H., 1999, "Fiscalité optimale et choix des instruments de taxation", in L'architecture des prélèvements en France : état des lieux et voies de réforme, Bourguignon F. et Bureau D., Rapport $\mathrm{du}$ CAE, La Documentation française, 83-92.

DME, 2005, Croissance et développement d'une économie insulaire - une étude des dynamiques de l'économie calédonienne, Rapport final AFD-IEOM, mai, 50 pages.

Ebrill L., Keen M., Bodin J-P., Summers V., 2002, "L'attrait de la taxe sur la valeur ajoutée", Finances \& développement, juin, pp. 44-47.

Fontaneau, 2001, "La TVA communautaire : Les principes", Fiscalité européenne et droit international des affaires, $\mathrm{n}^{\circ}$ 128, Cabinets FONTANEAU, 2001.

Houriez J-M., Roux V., 2001, "Vue d'ensemble des inégalités de revenus et de patrimoine", in Inégalités économiques, Atkinson T., Glaude M., Olier L. et Piketty T. Rapport du CAE, La Documentation française, 269-284.

IEOM, 2008, Rapport annuel de la balance des paiements de la Nouvelle-Calédonie, 30 pages.

ITSEE, 2000, Tableaux de l'économie calédonienne, 256 pages.

ISEE, 2007-a, Chiffres clés 2006, 11 juillet.

ISEE, 2007-b, Tableaux de l'économie calédonienne - Edition abrégée 2007, 147 pages.

ISEE, 2008, Tableaux de l'économie calédonienne - Edition abrégée 2008, 147 pages.

Keen M., Ligthart J. E., 2002, "Coordinating tariff reduction and domestic tax reform", Journal of International Economics 56, 489-507.

Keen M., Ligthart J. E., 2005, "Coordinating tariff reduction and domestic tax reform under imperfect competition", Review of International Economics, 13, 2, 385-390. 
Laborde D., 2007, "Panorama mondial des politiques commerciales", La Lettre du CEPPI, n² 267, mai, 4 pages.

Lamotte H., Saint Aubin B., 1999, "TVA et redistribution", in L'architecture des prélèvements en France : état des lieux et voies de réforme, Bourguignon F. et Bureau D., Rapport du CAE, La Documentation française, 109-121.

Malinvaud E., 1998, Les cotisations sociales à la charge des employeurs : analyse économique, Rapport du CAE, La Documentation française, 116 pages.

Marini Ph., 2006, Contribution présentée à la Société des Agriculteurs de France, 2 mai, 5 pages.

Perret C., 2002, Perspectives de développement pour la Nouvelle-Calédonie, PUG, Grenoble, 160 pages.

Ramsey F., 1927, "A contribution to the theory of taxation", Economic Journal, vol. 37, n 145, 47-61.

Salanié B., 1998a, "Un exemple de taxation optimale", in Fiscalité et redistribution, Bourguignon F., Rapport du CAE, La Documentation française, 87-90.

Salanié B., 1998b, "Incidence fiscale et fiscalité optimale à l'équilibre", in Fiscalité et redistribution, Bourguignon F., Rapport du CAE, La Documentation française, 91-95.

Ulla P., 2006, "Assessing Fiscal Risks through Long-term Budget Projections", OECD Journal on Budgeting, vol. 6, 1, 130-191.

\section{NOTES}

1. Cet article a bénéficié des commentaires de deux rapporteurs anonymes ainsi que de Bruno Boidin (USTL), Guy Agniel et Mathias Chauchat (UNC).

2. Le 5 mai 1998 a en effet été signé l'Accord de Nouméa, par le gouvernement français, le Rassemblement pour la Calédonie dans la République (RPCR) et le Front de Libération Nationale Kanak Socialiste (FLNKS). Cet Accord a ensuite été formalisé par une loi organique, en 1999. L'accord repousse à 2014 ou 2018 les référendums d'autodétermination. Pendant ces 20 ans la Nouvelle-Calédonie doit démontrer ses capacités de développement et, comme le précise le préambule de l'Accord de Nouméa, “(...) au terme d'une période de vingt années, le transfert à la Nouvelle-Calédonie des compétences régaliennes, l'accès à un statut international de pleine responsabilité et l'organisation de la citoyenneté en nationalité seront proposés au vote des populations intéressées. Leur approbation équivaudrait à la pleine souveraineté de la Nouvelle-Calédonie. »

3. À titre d'illustration, si l'IDH des pays de l'OCDE est supérieur à 0,9 (0,93 pour la France), les petits États insulaires sont très mal classés : le premier, Tonga, est $63^{\text {ème }}$, suivi par Samoa $\left(75^{\text {ème }}\right)$, Fidji $\left(81^{\text {ème }}\right)$. Il est surtout marquant de constater que le Vanuatu, seule ex-colonie française à être un pays océanien, a un IDH très faible : 0,57 (129 ème sur 177 pays classés).

4. Cette parité a été fixée par le gouvernement français en application du Protocole sur la France, annexé au Traité de Maastricht qui stipule que "la France conservera le privilège d'émettre des monnaies dans ses territoires d'outre-mer selon des modalités établies par sa législation nationale et elle sera seule habilitée à déterminer la parité du franc CFP. ». La parité du franc CFP est depuis 1999 de $1.000 \mathrm{~F}$. CFP pour 8,38 euros.

5. ISEE 2000 (p. 158). En 2007, le total des crédits des comptes revenus et transferts courants de la balance des paiements calédonienne se montait à 127 milliards de F. CFP (IEOM, 2008, p. 26).

6. Pour illustrer l'aspect dynamisant d'une TVA par rapport à la fiscalité douanière, il suffit de rappeler l'impact sur les inputs. Une entreprise voulant produire localement doit pour cela 
importer la quasi-totalité de ses inputs. Pour les recevoir elle doit payer les taxes douanières, préalablement à toute production (et même si les inputs se révélaient inutilisables ou étaient détruits entre le port et le lieu de stockage de l'entreprise), la fiscalité douanière correspond donc à une charge pour l'entreprise (ou un flux de sortie de trésorerie) avant toute activité. A contrario, la TVA n'est versée qu'après l'activité (et par rapport à la valeur ajoutée générée).

7. Les accords de l'OMC sont le socle du multilatéralisme commercial et constituent les règles juridiques de base pour le commerce international et la politique commerciale. Ils visent trois objectifs principaux : favoriser autant que possible la liberté des échanges ; poursuivre progressivement la libéralisation par voie de négociation; instituer un moyen impartial de règlement des différends.

8. Sur les restrictions quantitatives s'appliquent les articles VII, XI, XII et XX de l'Accord de 1947 (consolidé). Le 1 de l'article XI, «élimination générale des restrictions quantitatives », précise : "Aucune partie contractante n'instituera ou ne maintiendra à l'importation d'un produit originaire du territoire d'une autre partie contractante, à l'exportation ou à la vente pour l'exportation d'un produit destiné au territoire d'une autre partie contractante, de prohibitions ou de restrictions autres que des droits de douane, taxes ou autres impositions, que l'application en soit faite au moyen de contingents, de licences d'importation ou d'exportation ou de tout autre procédé". Cette interdiction générale des restrictions quantitatives est nuancée par le 2 dudit article. Mais il existe des conditions à remplir, que ne satisfait pas la Nouvelle-Calédonie. Or, lorsque la Nouvelle-Calédonie élabore son tarif douanier ou toute autre mesure visant à instaurer des restrictions quantitatives à l'importation (en dehors des exceptions expressément admises par les Accords et du cadre spécifique de la décision d'association des PTOM à la Communauté européenne), elle devrait respecter les engagements figurant dans lesdits instruments internationaux. Il apparaît donc que pour être juridiquement valable au regard des règles de l'OMC, toute restriction quantitative instaurée par la NouvelleCalédonie devrait entrer dans la catégorie des exceptions admises par les divers Accords sur le commerce des marchandises. Il convient, par ailleurs, de remarquer que la mise en place de telles mesures n'est pas une compétence unilatérale du Membre concerné, mais une attribution de la Conférence ministérielle de l'OMC (organe décisionnel composé de représentants de tous les Membres) qui décide d'une dérogation... Il semblerait que la France n'ait sollicité aucune dérogation en faveur de la Nouvelle-Calédonie.

9. Ainsi, R. J. Barro (1979) discute l'hypothèse d'équivalence ricardienne (selon laquelle l'effet des dépenses publiques sur l'économie est totalement indépendant de la façon dont sont financées les dépenses et donc du choix entre l'impôt ou l'emprunt, voire la création monétaire). Dans ce cadre, Barro estime que cette équivalence ne tient plus dès lors qu'on considère l'impact distorsif des taxes : la perte sociale générée par une hausse du taux d'imposition à une période, suivie d'une baisse équivalente à la période suivante, est supérieure à la perte sociale qui résulterait de deux variations nulles consécutives. Ainsi, il est alors optimal pour le gouvernement de maintenir un taux d'imposition constant dans le temps (« tax smoothing »). $\mathrm{H}$. Bohn (1990) qui analyse la structure optimale de la dette publique (sur données américaines) conclut que, dès lors qu'il existe des taxes distorsives, le gouvernement doit pratiquer un lissage des taux ("tax smoothing ») à la fois dans le temps et en fonction des états de la nature (chocs économiques).

10. Ainsi la Taxe de Base à l'Importation (TBI), au taux de 5\%, remplace la Taxe Spéciale, la Taxe de Consommation intérieure sur les produits Importés (TCI) remplace la Taxe de Consommation Intérieure, ainsi que la Taxe sur les Boissons Alcoolisées, la Taxe sur les Tabacs et la Taxe sur les Carburants, la Taxe Conjoncturelle pour la Protection de la Production Locale (TCPPL) remplace la Taxe Conjoncturelle sur les Produits Manufacturés et la Taxe sur les Bois.

11. Ce chiffre est relativement stable, puisqu'en 1999 les recettes douanières représentaient $24 \%$ de la valeur totale des importations, ce qui plaçait la Nouvelle-Calédonie au sixième rang mondial 
(après la Thaïlande, la Polynésie Française, la Mauritanie, la Libye et l'Inde, et avant la Tanzanie, le Malawi et la Tunisie).

12. L'analyse empirique montre que, toutes choses égales par ailleurs, les recettes de TVA augmentent avec la part du commerce international dans l'économie. (Banque Mondiale : http:// wbln0018.worldbank.org/html/smallstates.nsf/(attachmentweb)/1106172v1-les/ \$FILE/1106172v1-les.pdf).

13. Le taux zéro correspond à la situation dans laquelle le taux de la taxe appliquée aux ventes est nul, mais l'entrepreneur est autorisé à déduire la taxe payée en amont sur ses achats. Dans ce cas, l'entreprise dispose d'un droit à un remboursement intégral de la taxe payée sur ses achats. Dès lors que la TVA est conçue pour n'être qu'une taxe sur la consommation intérieure, les exportations sont imposées à un taux zéro et, par conséquent, quittent le pays sans avoir été grevées d'aucune TVA intérieure. Ce principe de destination constitue la norme internationale en matière de fiscalité indirecte, la taxe totale payée sur un produit étant déterminée par le taux perçu dans le pays de la vente finale et le montant de recettes revenant à ce pays. L'alternative au principe de destination est le principe d'origine, en vertu duquel la taxe est payée aux pays dans lesquels le produit est fabriqué et au taux en vigueur dans ceux-ci plutôt que là où il est consommé.

14. La TVA serait théoriquement à acquitter pour chaque opération taxable. Mais, dans un souci évident de simplification, les assujettis sont autorisés à liquider la TVA pour l'ensemble des opérations qu'ils réalisent au cours d'une période donnée, le mois généralement, ou, en fonction des pays, le trimestre ou même l'année pour les entreprises les plus modestes.

15. Étant donné qu'un impôt sur le chiffre d'affaires est perçu sur le produit des ventes indépendamment de la valeur ajoutée, la taxe collectée sur un produit donné reflétera le nombre d'étapes imposables dans la chaîne de sa production, entraînant un effet « en cascade » dans la charge fiscale. Cette situation incite les producteurs à délaisser les inputs taxés, pour limiter leur coût. Il y a donc une rentabilité sur le plan individuel, mais inefficience d'un point de vue social. De ce fait, les industries sont incitées à l'intégration verticale uniquement pour réduire leur charge fiscale, ce qui constitue une distorsion supplémentaire.

16. On trouve quelques indications dans les diverses études relatives aux frais de recouvrement de la TVA dans les pays de l'OCDE. Il a été estimé que les coûts d'administration de la TVA s'établissent à environ 100 dollars américains dans le meilleur des cas (environ 75 euros), par entrepreneur et par année. Quant au montant approximatif des coûts supportés par l'assujetti, il serait autour de 500 dollars américains par assujetti et par année (environ 360 euros). Ces chiffres sont supérieurs aux coûts équivalents dans les pays en développement, puisqu'ils contiennent une large part de coûts de main-d'œuvre.

17. L'expérience montre que nombre de pays ont eu tendance à fixer un seuil trop bas, se retrouvant ainsi confrontés à des difficultés considérables si les services fiscaux n'ont pas la capacité de gérer un grand nombre d'assujettis à la TVA (plus le seuil est fixé bas et plus il y a d'entreprises assujetties).

18. La TVA s'applique également dans trois DOM (La Réunion, la Guadeloupe et la Martinique) mais avec plusieurs particularités : des taux inférieurs à ceux de la métropole; des exonérations portant sur environ 8000 positions tarifaires ; la possibilité pour les entreprises de déduire de leur TVA collectée les montants non acquittés au titre des biens exonérés de TVA qu'ils immobilisent ou qu'ils incorporent dans leur production de biens et de services : la TVA Non Perçu Récupérable ou «TVA fictive » (pour un panorama de ce mécanisme, voir Avice et Cannard 2007).

19. Sur un plan comptable on définit la valeur ajoutée ainsi Valeur ajoutée = Marge commerciale + Production de l'exercice - Consommations de l'exercice en provenance de tiers. (La marge commerciale étant la différence : Ventes de marchandises - Coût d'achat des marchandises vendues). 
20. "L'enjeu, maintenant, c'est d'entreprendre une nouvelle réforme concernant, cette fois, les cotisations patronales. Il faut les calculer sur une assiette plus juste et plus favorable à l'emploi. (...) C'est essentiel pour sauvegarder un haut niveau de protection sociale dans une économie mondialisée.

Pour avancer dans ce sens, certains explorent la piste de la TVA sociale : baisser le coût du travail et augmenter le prix des produits importés, pour lutter contre les délocalisations. Une telle réforme est envisagée ou engagée dans des pays voisins. Mais il n'est pas question d'adopter brutalement un tel système, qui suppose, en toute hypothèse, un consensus national sur l'évolution des prix et des salaires. C'est pourquoi je proposerais de basculer une fraction des cotisations patronales sur une cotisation assise sur l'ensemble de la valeur ajoutée des entreprises." (Allocution de J. Chirac, Président de la République, à l'occasion des vœux aux forces vives - 5 janvier 2006).

21. Le corollaire attendu de la baisse du coût du travail est la diminution du travail au noir.

22. La TVAS est aussi moins injuste que les systèmes traditionnels de sécurité sociale. La CSVA serait aussi payée notamment par les touristes, par ceux qui dépensent leur épargne, alors que la sécurité sociale n'est payée que par ceux qui travaillent.

23. On peut considérer également que la TVAS est un moyen de rétablir une concurrence non faussée au niveau international. Le processus de mondialisation tend à supprimer toute barrière douanière entre marchés pourtant soumis à des conditions de production très inégales. Les pays se trouvent ainsi, contrairement aux règles affichées de l'orthodoxie libérale, et aux prescriptions même de l'OMC, soumis à une concurrence faussée (il n'y a pas de SMIC ni autres règles sociales internationales). C'est le problème dit du «dumping social » : ainsi un pays exploitant sa main-d'œuvre de façon indigne (enfants pas ou peu payés, etc.) y gagnera un avantage compétitif sur le coût du travail. Cet avantage compétitif serait effacé par la TVAS, puisque les importations y seraient soumises.

24. La CSG est un impôt comportant deux spécificités : elle est affectée, contrairement au principe d'universalité de l'impôt ; elle est affectée au financement de la protection sociale, comme les cotisations sociales.

25. Par rapport à une TVAS, le financement des cotisations sociales par la CSG aurait un autre avantage, dans le cas de la France, celui de contribuer au rapprochement des fiscalités des pays de l'Union européenne (Malinvaud 1998, p. 42).

26. $6,20 \%$ des revenus du chômage et $6,60 \%$ des retraites $(3,80 \%$ pour les personnes non assujetties à l'impôt sur le revenu) ; 8,20 \% des revenus du patrimoine et de placement ; 9,50\% des revenus des jeux.

27. La CAFAT (Caisse de Compensation des Prestations Familiales, des Accidents du Travail et de Prévoyance des travailleurs salariés de la Nouvelle-Calédonie) a été créée en 1958. Elle assure la gestion de cinq régimes de prestations sociales que sont les Accidents du travail, le Chômage, les Prestations Familiales, l'Assurance Maladie (RUAMM) et la Retraite. Concernant le financement des retraites, la Caisse Locale de Retraites de Nouvelle-Calédonie (CLR) est une caisse de retraite par répartition instituée au profit des fonctionnaires locaux.

28. Informations documentaires transmises par le cabinet de D. Leroux membre du Gouvernement de la Nouvelle-Calédonie de 2004 à 2006 (en charge notamment de la fiscalité).

29. De nombreux débats ont eu lieu en France sur cette question à partir de juin 2007, paraissant plutôt éloigner la perspective d'une prochaine instauration du mécanisme. En septembre 2007, le Secrétaire d'État chargé de la prospective et de l'évaluation des politiques publiques a remis au Premier Ministre un rapport complet sur la TVAS (Besson 2007), mais ne faisant pas de recommandation sur un calendrier de réforme.

30. Informations documentaires transmises par le cabinet de D. Leroux membre du Gouvernement de la Nouvelle-Calédonie de 2004 à 2006 (en charge notamment de la fiscalité).

31. Afin d'estimer le montant de TVA nécessaire pour compenser la suppression de la TSS, le montant de la TSS (au taux de 4\%) inscrite au budget 2006 avait été retenu, mais en adaptant ce chiffre en recalculant la TSS au taux de $5 \%$ sur une année entière. 
32. "Un besoin de financement d'environ 4 milliards de F. CFP serait une bonne base de travail pour la seule couverture des déficits annuels des régimes sociaux, ce qui mécaniquement produit une hypothèse de taux (de CSG) autour de 1,5\% " (Chauchat 2006, p. 84).

33. Source : www.cafat.nc.

34. Le déficit de la CLR était de 2,13 milliards F. CFP en 2005. En 2006, le déficit s'est monté à 647 millions F. CFP (ISEE 2008, p. 82).

35. Le Président de la République N. Sarkozy a annoncé réfléchir à l'instauration d'une TVAS, déclarant en janvier 2007 « préférer taxer la consommation plutôt que l'emploi » (Les échos, 16/01/07). 36. C'est-à-dire en comparant la TVA non pas à la fiscalité douanière actuelle, mais à une fiscalité douanière réformée se substituant à l'ensemble des taxes que devrait remplacer la TVA. En revanche, si on se place dans l'optique d'un développement économique, alors la balance penchera progressivement pour une TVA même normale.

37. Comme l'explique Malinvaud: "Supposons qu'une diminution indifférenciée du taux des cotisations patronales soit introduite simultanément avec une augmentation compensatoire du taux de la TVA. Une telle décision devrait immédiatement déclencher un ensemble de réactions, tantôt simultanées tantôt s'effectuant en cascade. Elles concerneront les prix hors taxes, les prix taxes comprises, les coûts, les transferts, les anticipations, etc. Suivre précisément cet ensemble de réactions supposerait une connaissance fine des formes de la concurrence, des modalités des décisions salariales, des rigidités des prix et des salaires, des règles de révision des transferts, etc. Cela d'autant plus que les situations varient, d'un bien à un autre, d'une branche à une autre, d'un transfert à un autre, et d'autant plus aussi que le contexte dans lequel ces multiples réactions se produiront sera lui-même influencé par elles, notamment s'il y a des effets favorables à l'emploi. Puisque nous ne disposons pas de la connaissance fine qui serait requise pour un suivi précis au delà de quelques trimestres, nous devons faire appel à notre jugement et schématiser en portant l'attention autant sur les prix et coûts relatifs que sur les effets de volume. " (Malinvaud 1998, p. $40)$.

38. $8 \% \div 36$ 틀 $92=20,44 \%$.

39. L'impact de la TVAS sur les prix dépendrait, pour chaque secteur, de la part représentée par les salaires dans le coût de production. Plus cette part serait élevée et plus le prix de vente TTC serait mécaniquement diminué par la substitution de la TVAS aux cotisations patronales. La part des salaires dépend des secteurs, les prix diminueraient pour certains secteurs et augmenteraient pour d'autres. C'est au niveau de l'économie dans son ensemble que l'effet serait neutre sur les prix, pour un taux de TVAS calibré à cet effet (et qui nécessiterait des simulations économiques poussées).

40. Même si les deux cas sont suffisamment atypiques pour ne pas être similaires, il n'y a pas vraiment d'autres territoires ou pays plus adaptés pour une comparaison (aspect petite économie insulaire, importance des transferts métropolitains, etc.)

\section{RÉSUMÉS}

L'économie calédonienne est emplie de contradictions: retard de développement, protectionnisme sans TVA, émancipation politique (Accord de Nouméa) mais faible taux de couverture et importants transferts de la métropole... Cet article analyse, d'un point de vue fiscal, la soutenabilité du système néo-calédonien actuel: comment peut-il perdurer sans crise structurelle? Quelle réforme fiscale proposer ? La section 1 justifie la réforme. La section 2 décrit 
le système économico-commercial calédonien. La section 3 présente les principes de la TVA; la section 4 ceux de la TVA sociale. La section 5 analyse la substitution d'une fiscalité douanière par une TVA fiscale ou sociale.

New-Caledonia's economy concentrates many contradictions: under development, protectionism, no VAT, political emancipation but high financial transfers from France... This paper analyses, from a taxation point of view, the sustainability of the current New-Caledonian economic system: how could this system persist without any structural crisis? Which tax reform should be proposed? The section 1 explains why tax reform is necessary. The section 2 describes New-Caledonia's economic and commercial system. The sections 3 and 4 present the principles of the VAT and of the social VAT. The section 5 analyses the opportunity to change New-Caledonia's customs tax for a VAT or a social VAT.

\section{INDEX}

Mots-clés : développement, tarifs douaniers, réforme fiscale, TVA

Keywords : development, tarifs, tax reform, value-added tax

\section{AUTEUR}

\section{GAËL LAGADEC}

Gaël Lagadec est Maître de conférences à l'Université de la Nouvelle-Calédonie et membre du LARJE (http://larje.univ-nc.nc). Ses thèmes de recherche sont l'économie du développement et la protection commerciale endogène. 